\title{
Consequences of Asteroid Fragmentation during Impact Hazard Mitigation
}

\author{
J.P. Sanchez* \\ University of Strathclyde, Glasgow, Scotland G1 1XH, United Kingdom \\ M.Vasile ${ }^{\dagger}$ and G. Radice \\ University of Glasgow, Glasgow, Scotland G12 8QQ, United Kingdom
}

\begin{abstract}
The consequences of the fragmentation of an Earth threatening asteroid due to an attempted deflection are examined in this paper. The minimum required energy for a successful impulsive deflection of a threatening object is computed and compared to the energy required to break-up a small size asteroid. The results show that the fragmentation of an asteroid that underwent an impulsive deflection, such as a kinetic impact or a nuclear explosion, is a very plausible event. A statistical model is used to approximate the number and size of the fragments as well as the distribution of velocities at the instant after the deflection attempt takes place. This distribution of velocities is a function of the energy provided by the deflection attempt, while the number and size of the asteroidal fragments is a function of the size of the largest fragment. The model takes also into account the gravity forces that could lead to a re-aggregation of the asteroid after fragmentation. The probability distribution of the pieces after the deflection is then propagated forward in time until the encounter with the Earth. A probability damage factor (i.e., expected damage caused by a given size fragment multiplied by its impact probability) is then computed and analyzed for different plausible scenarios, characterized by different levels of deflection energies and lead times.
\end{abstract}

\section{Nomenclature}

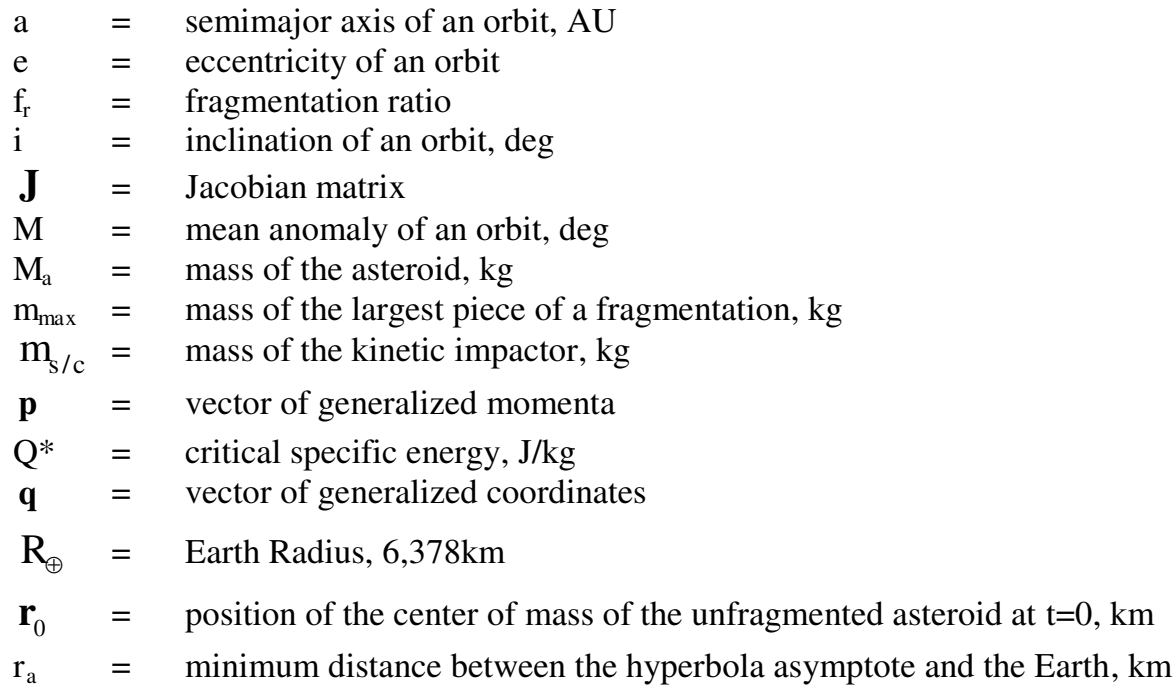

\footnotetext{
${ }^{*}$ Research Fellow, Department of Mechanical Engineering, James Weir Building; jpau.sanchez@strath.ac.uk.

${ }^{\dagger}$ Senior Lecturer, Department of Aerospace Engineering, James Watt South Building, AIAA Senior Member. m.vasile@aero.gla.ac.uk

* Senior Lecturer, Department of Aerospace Engineering, James Watt South Building, AIAA Member. g.radice@aero.gla.ac.uk
} 


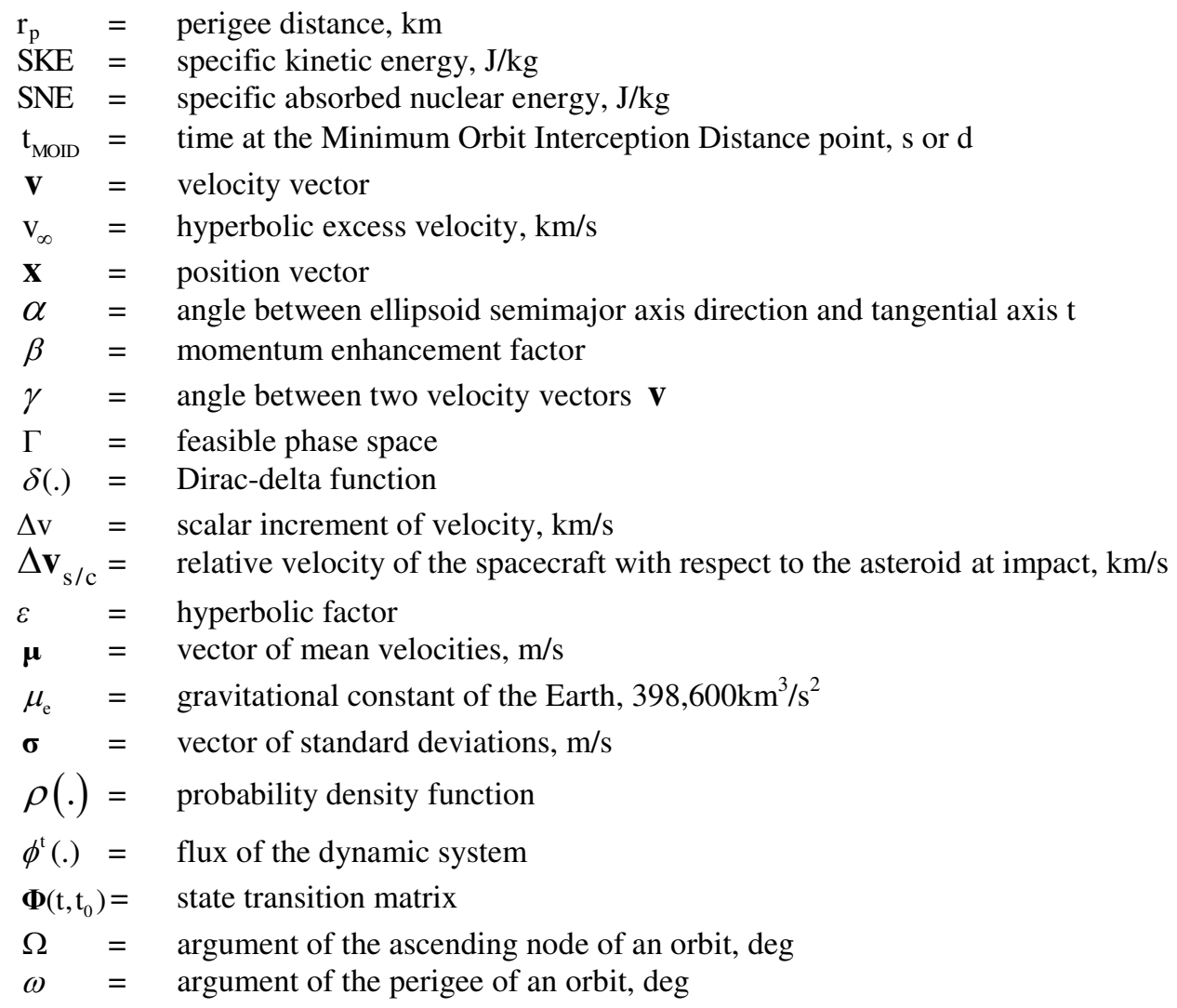

\section{Introduction}

The threat that asteroids pose to life on Earth has for long been acknowledged ${ }^{[1]}$. Many techniques to deviate threatening asteroids have been proposed in the last three decades. Some of these techniques propose the application of a very low acceleration on the asteroid during long periods of time, while others use a high speed impact or an explosion (e.g., nuclear warhead) to produce an impulsive change in linear momentum. If an impulsive deviation technique is applied to an asteroid, and the energy delivered by the deviation method is above a limit threshold ${ }^{[2-4]}$, a catastrophic fragmentation, i.e., fragmentation such that the largest fragment contains less than half the mass of the original asteroid, is likely to occur.

Plenty of studies have classified, evaluated and compared the existing techniques in terms of deviation efficiency $^{[5-9]}$, but little has been done on the analysis of a possible fragmentation ${ }^{[10]}$. This paper tries to discern the feasibility of deflecting asteroids using impulsive mitigation techniques such as kinetic impactors and nuclear interceptors, examining also the consequences of a catastrophic fragmentation in terms of potential damage to Earth. In particular, we consider the minimum level of energy (collisional energy) required to deviate an asteroid by a distance that ensures a successful deflection, even considering the hyperbolic trajectory that the asteroid will follow when approaching the Minimum Orbit Interception Distance (MOID) from the Earth. This minimum level of collisional energy is strongly dependent on the lead time, or time prior to the impact with the Earth at which the deflection maneuver is applied. The level of collisional energy is then compared with the predicted specific energy required to completely fracture an asteroid, which can be inferred from experimental work in impact fragmentation $^{[11]}$ and numerical modeling ${ }^{[3]}$. As will be shown in the paper, for some lead times the collision energy required for an impulsive deviation technique can rise well above the theoretical catastrophic fragmentation limit.

As a consequence, an asteroid that underwent an impulsive deflection attempt may fragment in an unpredictable number of pieces having different mass and velocity. The number of fragments and its size distribution can be described with fairly good approximation by a power law ${ }^{[11-13]}$, while the distribution of velocity of the fragments produced by the catastrophic break-up is described by a Gaussian function with a standard velocity deviation varying with fragment size. This paper assumes homogenous distribution of the translational kinetic energy among all the fragments, or equipartition effect ${ }^{[14]}$, with a certain amount of energy loss by fragmentation-related processes, such as breaking or melting. Approximating all fragments as departing from the centre of mass of the unshattered asteroid, the velocity associated with each piece of the asteroid will uniquely determine its future trajectory. 
The fragmentation dynamical model in this paper assesses the effect of the gravitational attraction of the largest fragment resultant from the catastrophic disruption. In fact, if the initial relative velocity of the largest pieces is not high enough, partial or total re-aggregation of the cloud of fragments, forming a rubble-pile asteroid, may occur. The evolution of the cloud of fragments with an initial relative velocity high enough to avoid re-aggregation is computed through the use of Liouville's theorem for Hamiltonian systems and considering a two-body dynamical model.

The risk that a fragmented asteroid may pose to Earth is quantitatively evaluated by computing the equivalent statistical surface area hit by the fragments, referred here as damage probability. It is known that asteroidal bolides larger than a few tens of meters in diameter are already able to cause significant damage to the Earth surface due to the sudden blast produced by the dissipation of the bolide when crossing the Earth atmosphere, e.g. Tunguska impact $^{[15]}$. Bolides above $150-200 \mathrm{~m}$ in diameter ${ }^{[16 ; 17]}$, instead, reach the Earth surface producing cratering events and, if falling into the sea, dangerous tsunamis ${ }^{[18]}$.

The paper considers two possible break-up scenarios: the fragmentation being the desired outcome of the deviation strategy or the undesired product of a mitigation mission. Therefore, we include in the analysis deflection attempts with a broad range of collisional energies, varying from $100 \mathrm{~J} / \mathrm{kg}$ to $5000 \mathrm{~J} / \mathrm{kg}$ which includes specific energies above and below the range of energies considered here as possible fragmentation limits ${ }^{[2-4]}$.

\section{Fragmentation Energy}

In order to assess the likelihood of a fragmentation outcome from an impulsive mitigation technique, the asteroid resistance to fragmentation needs first to be estimated. The critical specific energy $Q^{*}$ is defined as the energy per unit of mass necessary to barely catastrophically disrupt an asteroid ${ }^{[3]}$; an asteroid is barely catastrophically disrupted when the mass of the largest fragment of the asteroid is half the mass of the original asteroid, or in other words, the remaining mass of the original asteroid is half the initial mass. If $f_{r}$ is the fragmentation ratio, defined as:

$$
\mathrm{f}_{\mathrm{r}}=\frac{\mathrm{m}_{\max }}{\mathrm{M}_{\mathrm{a}}}
$$

where $\mathrm{m}_{\max }$ is the mass of the largest fragment and $\mathrm{M}_{\mathrm{a}}$ the initial mass of the asteroid, then a catastrophic fragmentation is defined as a fragmentation where $\mathrm{f}_{\mathrm{r}}<0.5$. 


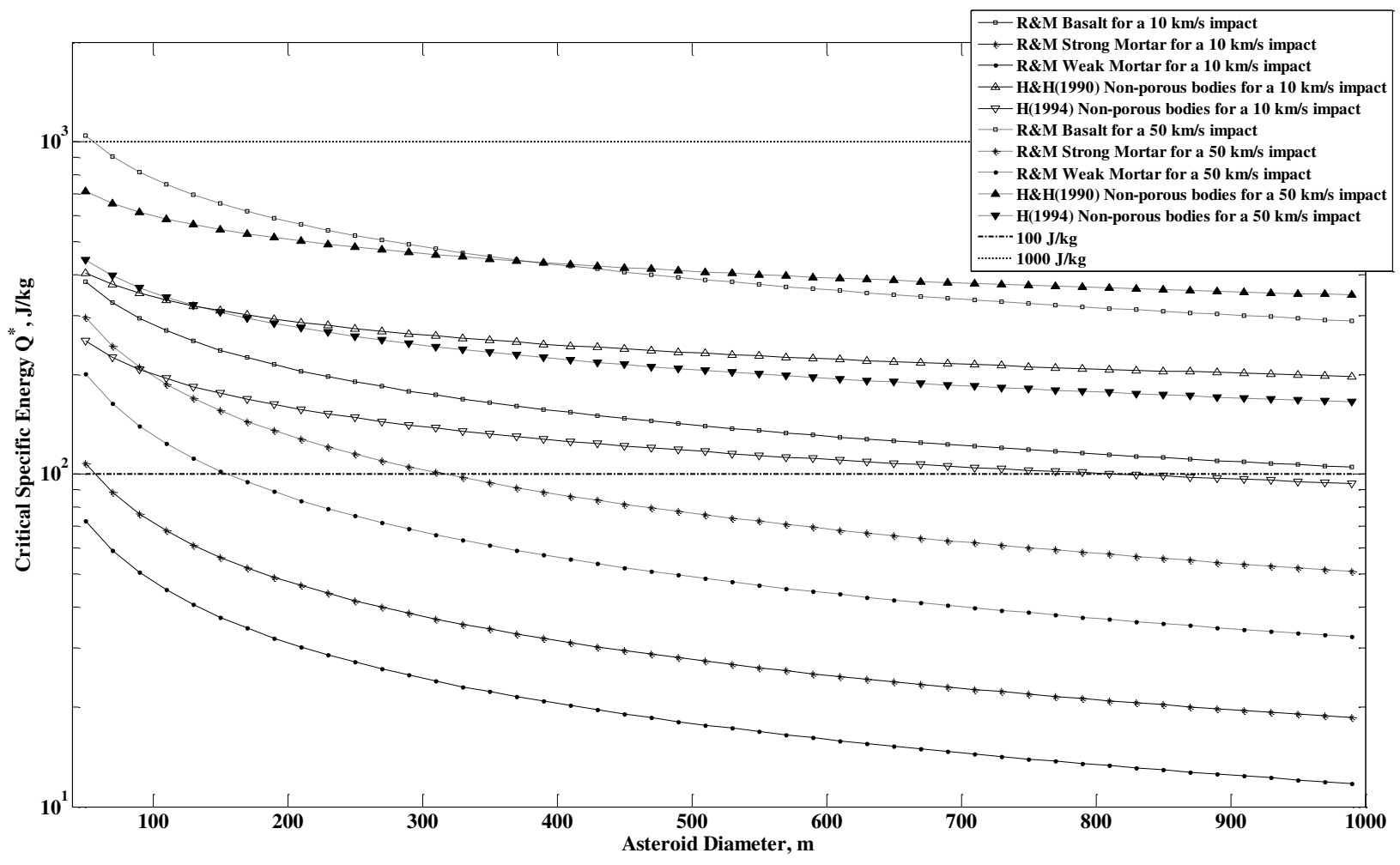

Fig. 1 Critical Specific Energy Q* for barely catastrophically disrupting asteroids with a diameter ranging from $40 \mathrm{~m}$ to $1 \mathrm{~km}$, calculated using the work of Ryan and Melosh $(\mathrm{R} \& H)^{[3]}$, Housen and Holsapple(H\&H(1990) $)^{[4]}$ and Holsapple ${ }^{[19]}$.

This paper addresses the issue of fragmentation of small to medium size asteroids. These are celestial objects ranging from $40 \mathrm{~m}$ to $1 \mathrm{~km}$ in diameter and constitute the main bulk of the impact threat. Small objects in this range rely only on their material strength properties to avoid break up, while for large objects gravity plays a fundamental role ${ }^{[13]}$. Asteroids smaller than $40 \mathrm{~m}$ in diameter are expected to dissipate at a high altitude in the Earth atmosphere ${ }^{[16]}$, thus nothing smaller than $40 \mathrm{~m}$ will be included in the analysis. On the other hand, the survey of objects with a diameter larger than $1 \mathrm{~km}$ is believed to be almost complete, therefore only the remaining small not discovered asteroids pose a threat ${ }^{[20]}$.

The uncertainty associated with the description of the fragmentation process is clear if one looks at the different scaling laws in the literature ${ }^{[13]}$. Furthermore, the exact value of $Q^{*}$ depends on a number of factors, such as the composition and structure of the asteroid or the velocity and the size of the impactor. For the sake of analysis, in this paper, a complete and exact description of the fragmentation process is not required and an approximate estimate of the value of the critical specific energy $Q^{*}$ is sufficient. The work of Ryan and Melosh ${ }^{[3]}$ and Housen and Holsapple ${ }^{[4 ; 19]}$ provided the necessary tools to understand and approximate the qualitative limits of the critical specific energy $Q^{*}$ for the range of studied asteroids. Fig. 1 shows the critical specific energy $Q^{*}$ for asteroids ranging from $40 \mathrm{~m}$ to $1 \mathrm{~km}$ diameter, computed by using the scaling laws provided by the aforementioned authors.

In the light of the results shown in Fig. 1, two general qualitative limits were drawn: one at $1000 \mathrm{~J} / \mathrm{kg}$ and a second at $100 \mathrm{~J} / \mathrm{kg}$. The upper fragmentation limit at $1000 \mathrm{~J} / \mathrm{kg}$ is above any of the specific energies $\mathrm{Q}^{*}$ expected from the scaling laws in Fig. 1. including those of basalt strength from Ryan and Melosh ${ }^{[3]}$ and non-porous rocky bodies from Housen and Holsapple ${ }^{[4]}$. Even in some cases, this upper limit is more than one order of magnitude above the predicted $\mathrm{Q}^{*}$. Hence, for the studied range of diameters, the limit at $1000 \mathrm{~J} / \mathrm{kg}$ is here considered as a fated catastrophic fragmentation. On the other hand, the lower fragmentation limit at $100 \mathrm{~J} / \mathrm{kg}$ is an approximate mean energy level among all the critical energies Q* predicted by Fig. 1, and more importantly, the $100 \mathrm{~J} / \mathrm{kg}$ limit is, in general, above the four predicted $Q^{*}$ using Ryan and Melosh ${ }^{[3]}$ 's Mortar strengths. If asteroids have the tensile strength of "rubble piles", as the rotational state of small asteroids seems to indicate ${ }^{[21]}$, the scaling laws for mortar tensile strength from Fig. 1 may be a good approximation. Hence, the $100 \mathrm{~J} / \mathrm{kg}$ limit may be considered as a reasonable fragmentation limit according to the results of Ryan and Melosh ${ }^{[3]}$. 


\section{Near Earth Objects (NEO) Deflection Requirements}

In order to compute the minimum deflection required to deviate a threatening asteroid, we will need to define the minimum distance that an asteroid needs to be shifted in order to miss the Earth. Since the threatening asteroid will follow a hyperbolic approach at the proximity of the Earth, the minimum distance of one Earth radius $\mathrm{R}_{\oplus}$ will need to be corrected in order to account for the gravitational pull of the Earth in its final approach. This correcting factor is:

$$
\varepsilon=\frac{\mathrm{r}_{\mathrm{a}}}{\mathrm{r}_{\mathrm{p}}}=\sqrt{1+\frac{2 \mu_{\mathrm{e}}}{\mathrm{r}_{\mathrm{p}} \mathrm{v}_{\infty}^{2}}}
$$

where $r_{a}$ is the minimum distance between the hyperbola asymptote and the Earth, i.e., focus of the hyperbola, $r_{p}$ is perigee distance, which is fixed to $\mathrm{R}_{\oplus}$ (minimum distance to avoid collision without considering the atmosphere altitude), $\mu_{\mathrm{e}}$ is the gravitational constant of the Earth and $\mathrm{v}_{\infty}$ the hyperbolic excess velocity. Note that the correcting factor only depends on the hyperbolic excess velocity of the threatening object.

As will be seen later, the analysis carried out in this paper is very sensitive to the orbital parameters of the asteroid. The three different test cases summarized Table 1 are therefore chosen in order to provide a better insight to the problem. Apophis is clearly an interesting test case, since it is the most renowned asteroid among those posing a noticeable threat to Earth. On the other hand, the Aten-case and Apollo-case were created from two sets of 100 asteroids belonging to the Aten and Apollo groups. The asteroids in the sets, were taken from the list of the most dangerous Earth Crossing Asteroid in the NASA's NEO program database ${ }^{\S}$, and while the semimajor axis a, eccentricity e and inclination i were generated using the mean of these variables from the aforesaid lists, the angular Keplerian elements, $\Omega, \omega$ and $\mathrm{M}_{0}$, were modified such that the MOID for a fixed collision date was minimal.Table 1 . shows the six Keplerian elements, the epoch of those elements, the $t_{\text {MOID }}$ or time of the minimum interception distance that is used as the virtual impact, the hypebolic factor $\varepsilon$, the impact velocity and the mass of each test case.

Table 1. Summary of the orbital characteristics of the three cases used in this work

\begin{tabular}{|c|c|c|c|c|c|c|c|c|c|c|c|}
\hline & $\begin{array}{c}\mathrm{a} \\
{[\mathrm{AU}]}\end{array}$ & $\mathrm{e}$ & $\begin{array}{c}\mathrm{i} \\
{[\mathrm{deg}]}\end{array}$ & $\begin{array}{c}\Omega \\
{[\mathrm{deg}]}\end{array}$ & $\begin{array}{c}\omega \\
{[\mathrm{deg}]}\end{array}$ & $\begin{array}{c}\mathrm{M}_{0} \\
{[\mathrm{deg}]}\end{array}$ & $\begin{array}{l}\text { Epoch } \\
\text { [MJD] }\end{array}$ & $\begin{array}{l}\mathrm{t}_{\mathrm{MOID}} \\
\text { [MJD] }\end{array}$ & $\varepsilon$ & $\begin{array}{c}\mathrm{v} \\
\text { impact } \\
{[\mathrm{km} / \mathrm{s}]}\end{array}$ & $\begin{array}{c}\mathrm{M}_{\mathrm{a}} \\
{[\mathrm{kg}]}\end{array}$ \\
\hline Apophis & 0.922 & 0.191 & 3.331 & 204.5 & 126.4 & 222.3 & 53800.5 & 62240.3 & 2.16 & 12.62 & $2.7 \times 10^{10}$ \\
\hline $\begin{array}{l}\text { Aten- } \\
\text { case }\end{array}$ & 0.875 & 0.313 & 7.828 & 259.9 & 50.65 & 97.21 & 62481.0 & 62182.1 & 1.52 & 14.85 & $5 \times 10^{10}$ \\
\hline $\begin{array}{l}\text { Apollo- } \\
\text { case }\end{array}$ & 1.706 & 0.518 & 10.70 & 266.8 & 121.2 & 18.09 & 62488.0 & 62488.0 & 1.29 & 17.78 & $5 \times 10^{10}$ \\
\hline
\end{tabular}

It can be noted, in Table 1, that Apophis has the largest hyperbolic factor of the three; this is due to the resemblance of its orbit to the Earth orbit. The more an orbit resembles to the Earth orbit, the lower the relative velocity at the encounter will be and, clearly, this makes the asteroid more susceptible to be affected by the Earth gravity, since it will spend more time in close encounter. For example, a relative velocity of $0 \mathrm{~km} / \mathrm{s}$ has an infinite hyperbolic factor and a minimum impact velocity of $11.18 \mathrm{~km} / \mathrm{s}$, which is the theoretical parabolic escape velocity. The opposite is also true; the more an orbit differs from the Earth orbit, the higher the relative velocity at encounter and the lower the hyperbolic factor. Therefore, Apollo-case is the one carrying the highest amount of kinetic energy, while Apophis is the most prone to "fall" into earth, i.e., largest hyperbolic factor.

\section{A. Minimum Change in Velocity}

Once the minimum distance to avoid collision is set (i.e., $\varepsilon \cdot \mathrm{R}_{\oplus}$ ), the minimum change of velocity to provide a safe deflection can be calculated.Fig. 2 presents the necessary change of velocity within an interval spanning 20 years before the hypothetical impact at time $t_{\text {MOID }}$ to deviate the three test cases. The minimum change of velocity required to deviate an object by a given distance from its initial orbit is here computed by means of proximal motion equations expressed as a function of the variation of the orbital elements. The variation of the orbital elements was

\footnotetext{
${ }^{\S}$ http://neo.jpl.nasa.gov/
} 
then computed with Gauss' planetary equations (see Vasile et al. ${ }^{[22]}$ for further details on the deflection formulas used in this paper).

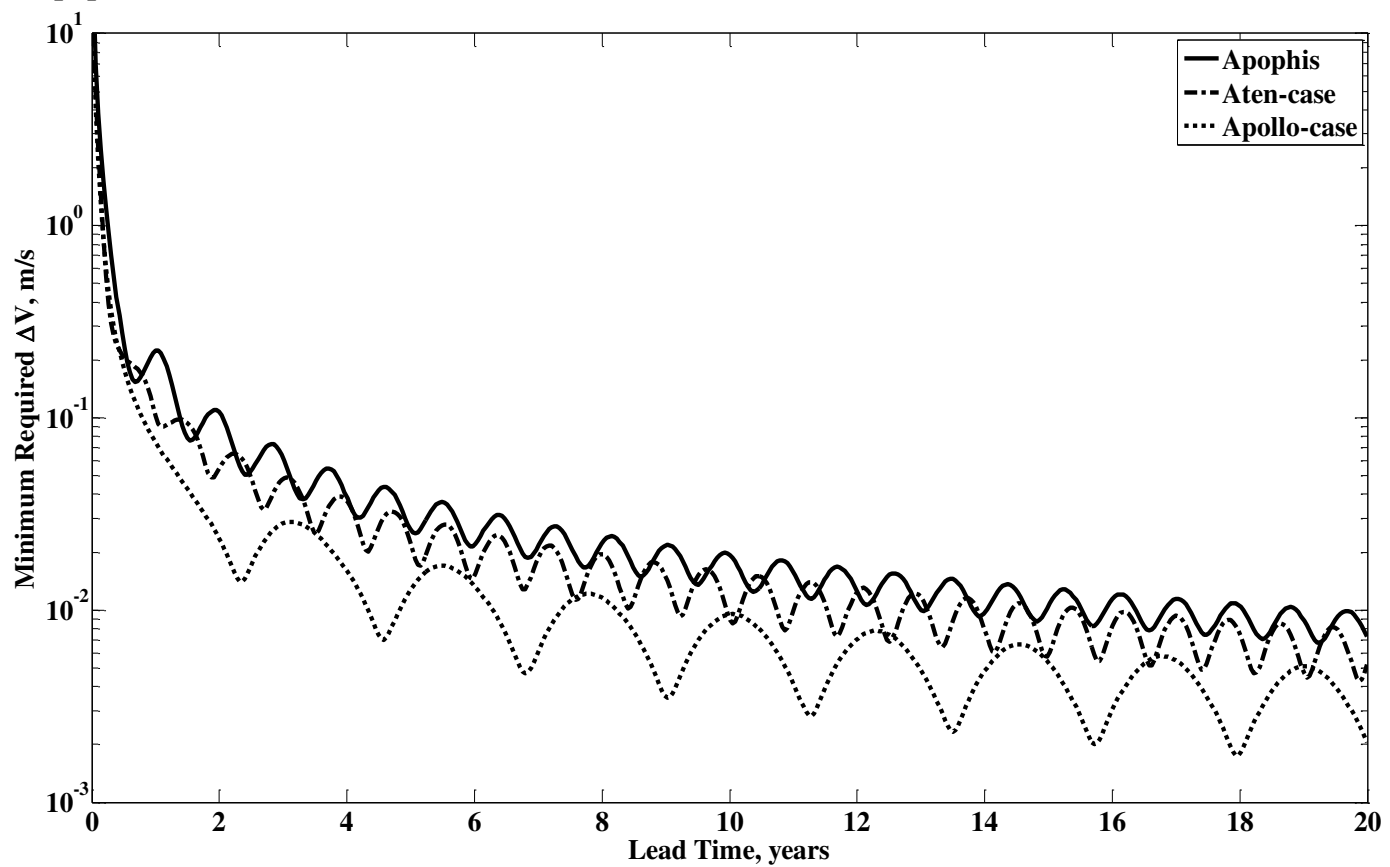

Fig. 2 Minimum required $\Delta \mathrm{v}$ for a $\varepsilon \cdot \mathrm{R}_{\oplus}$ deflection.

The minimum required $\Delta \mathrm{v}$ in Fig. 2 were computed using the hyperbolic factor $\varepsilon$ particular to each case. A very distinctive feature of Fig. 2 is the oscillatory behavior of the minimum required $\Delta \mathrm{V}$ to deflect the three test cases. This sinusoidal evolution repeats with the orbital period of the asteroid and its amplitude is a function of both the magnitude of the orbital velocity and the variation of the orbital velocity along a complete orbit. Each minimum occurs at the point where the asteroid is moving at its highest speed, thus its perihelium, and so, it is more vulnerable to changes in its orbital period.

\section{B. Kinetic Impactor and Nuclear Interceptor}

Many of the mitigation techniques described in the literature ${ }^{[5-9]}$ could provide the necessary change of velocity to ensure that a threatening object misses the Earth, but only impulsive mitigation actions can provide quasiinstantaneous specific energies of the order of the Critical Energy Q* from Fig. 1. Hence, deflection strategies such as kinetic impactor and nuclear interceptor could trigger a catastrophic outcome as a result of a deviation attempt. The remaining of this section will briefly review the main features of these two mitigations strategies, more comprehensive description can be found in other work by the authors ${ }^{[9 ; 23]}$.

The Kinetic Impactor is the simplest concept for asteroid hazard mitigation: the asteroid's linear momentum is modified by ramming a mass into it. The impact is modeled as an inelastic collision resulting into a change in the velocity of the asteroid multiplied by a momentum enhancement factor ${ }^{[24]}$. This enhancement is due to the blast of material expelled during the impact, although if the asteroid undergoes a fragmentation process after the impact, the enhancement factor should be considered 1, since all the material is included in the fragmentation process. Accordingly, the variation of the velocity of the asteroid $\Delta \mathbf{v}_{\mathrm{a}}$ due to the impact is given by:

$$
\Delta \mathbf{v}_{\mathrm{a}}=\beta \frac{\mathrm{m}_{\mathrm{s} / \mathrm{c}}}{\left(\mathrm{M}_{\mathrm{a}}+\mathrm{m}_{\mathrm{s} / \mathrm{c}}\right)} \Delta \mathbf{v}_{\mathrm{s} / \mathrm{c}}
$$

where $\beta$ is the momentum enhancement factor, $\mathrm{m}_{\mathrm{s} / \mathrm{c}}$ is the mass of the kinetic impactor, $\mathbf{M}_{\mathrm{a}}$ is the mass of the asteroid and $\Delta \mathbf{v}_{\mathrm{s} / \mathrm{c}}$ is the relative velocity of the spacecraft with respect to the asteroid at the time when the mitigation attempt takes place.

Knowing the minimum change of velocity required for a deflection (see Fig. 2), Eq.(3) can be used to compute the Specific Kinetic Energy (SKE) that an asteroid would have to absorb from a kinetic impactor mission attempting to modify its trajectory: 


$$
\mathrm{SKE}=\frac{1}{2} \frac{\mathrm{m}_{\mathrm{s} / \mathrm{c}} \Delta \mathbf{v}_{\mathrm{s} / \mathrm{c}}{ }^{2}}{\mathrm{M}_{\mathrm{a}}}=\frac{1}{2} \frac{\left(\mathrm{M}_{\mathrm{a}}+\mathrm{m}_{\mathrm{s} / \mathrm{c}}\right)^{2}}{\beta^{2} \cdot \mathrm{M}_{\mathrm{a}} \cdot \mathrm{m}_{\mathrm{s} / \mathrm{c}}} \Delta \mathbf{v}_{\mathrm{a}}^{2}
$$

Fig. 3 presents an example of the Specific Kinetic Energy (SKE) as a function of the lead time that a kinetic impactor should apply to the asteroid in order to provide the $\Delta \mathbf{v}_{\mathrm{a}}$ required in Fig. 2. The impactor mass $\mathrm{m}_{\mathrm{s} / \mathrm{c}}$, for this example, was set to $5,000 \mathrm{~kg}$ and the impact velocity $\Delta \mathbf{v}_{\mathrm{s} / \mathrm{c}}$ was calculated expecting and enhancement factor $\beta$ equal to $2^{[9]}$. Note also that, for a given delta-velocity $\Delta \mathbf{v}_{\mathrm{a}}$, the SKE will vary with the kinetic impactor mass $\mathrm{m}_{\mathrm{s} / \mathrm{c}}$, thus, an example with higher impact mass will provide a lower value of SKE. The two aforementioned fragmentation limits of $1000 \mathrm{~J} / \mathrm{kg}$ and $100 \mathrm{~J} / \mathrm{kg}$ are also superposed on the figure. In general terms, the SKE needed for very short warning times ( $<2$ years) is clearly above the fragmentation limits, only for very long warning times ( $>10$ years) the energy required for a kinetic deflection begins to move below the lower limit threshold.

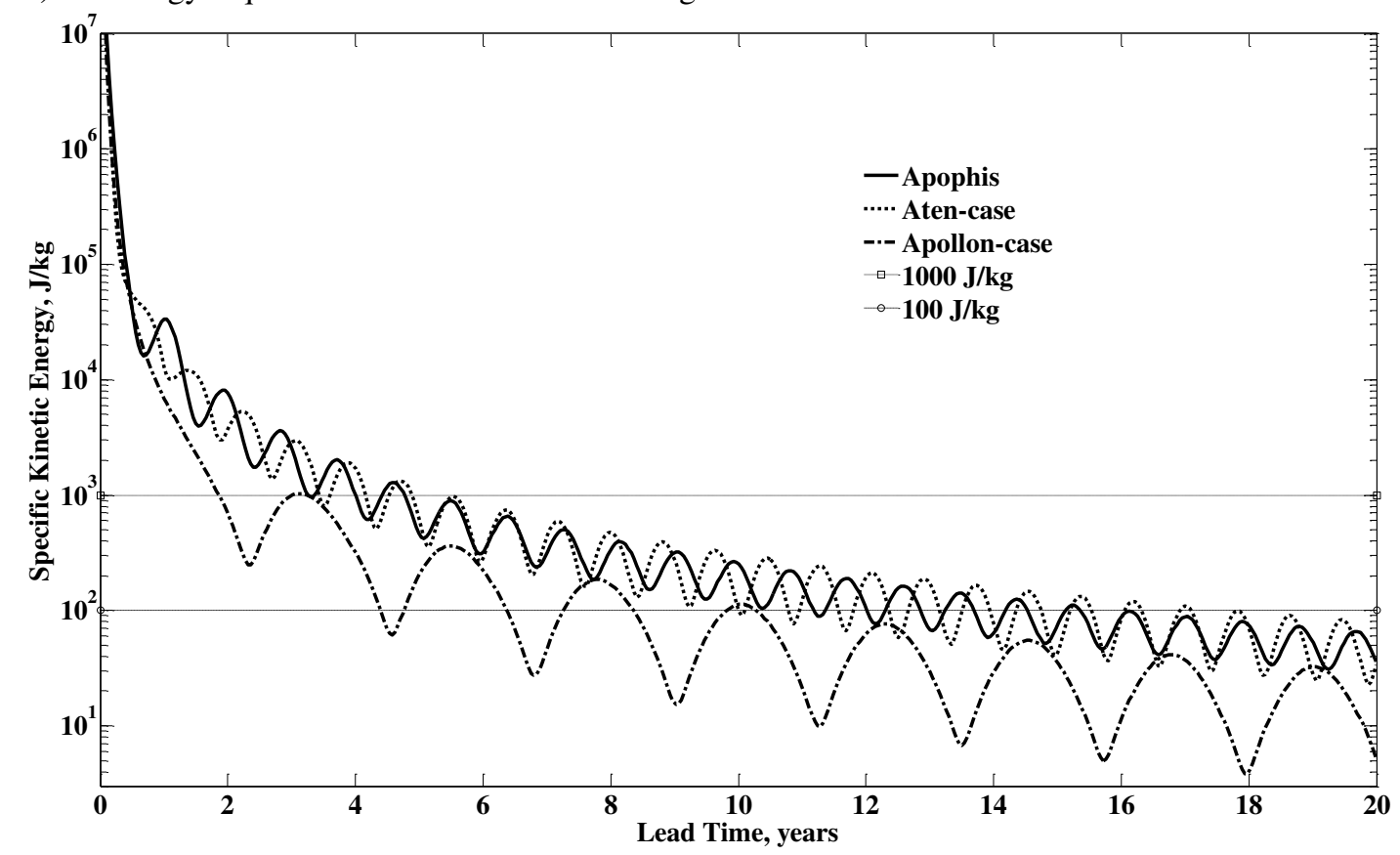

Fig. 3 Minimum SKE required for a deflection mission with $5,000 \mathrm{~kg}$ of impact mass as a function of the lead time.

It should also be noted that a kinetic impactor may require unrealistic impact velocities $\Delta \mathbf{v}_{\mathrm{s} / \mathrm{c}}$ to provide very large SKE. For example, in order for a kinetic impactor to deliver collisional energies greater than $1000 \mathrm{~J} / \mathrm{kg}$ it would need an impact mass of more than 50 tons and relative velocity larger than $50 \mathrm{~km} / \mathrm{s}$. Considering retrograde trajectories, impact velocities of at least $60 \mathrm{~km} / \mathrm{s}$ are possible ${ }^{[25]}$ even without using advanced propulsion concepts $^{[26]}$. Therefore, assuming impact velocities close to $50 \mathrm{~km} / \mathrm{s}$ only a few tons of impact mass would be required to provide a collisional energy on the order of $100 \mathrm{~J} / \mathrm{kg}$.

The Nuclear Interceptor strategy considers a spacecraft carrying a nuclear warhead and intercepting the asteroid. The model used in this study, fully described in Sanchez et al. ${ }^{[9]}$, is based on a stand-off configuration over a spherical asteroid, i.e., the nuclear device detonates at a given distance from the asteroid surface. The energy released during the explosion is carried mainly by X-rays, neutrons and gamma radiation that are absorbed by the asteroid surface. We assumed that the nuclear device detonates at the optimal stand-off distance found in Sanchez et al. ${ }^{[9]}$, which corresponds to an irradiation of only $3.5 \%$ of the total asteroid surface. As a consequence, it is assumed that the sudden irradiation of such a small spot would induce a stress wave that could trigger not only the surface material ablation but also the fragmentation of the whole body. Note that this is not the general expected outcome of a stand-off explosion as a larger irradiated area can reduce the transmitted shocks. However, the point here is not to demonstrate the likelihood of a fragmentation due to a nuclear explosion but rather to investigate the consequences of such a fragmentation should the nuclear explosion has an outcome comparable to a high velocity impact. The 
Specific absorbed Nuclear Energy (SNE) is defined here as the portion of the energy released that is radiated over the asteroid divided by the mass of the asteroid. Figure 4 shows the SNE function of lead time that a nuclear interceptor should apply to deflect a threatening asteroid with a delta velocity as specified in Fig. 2.

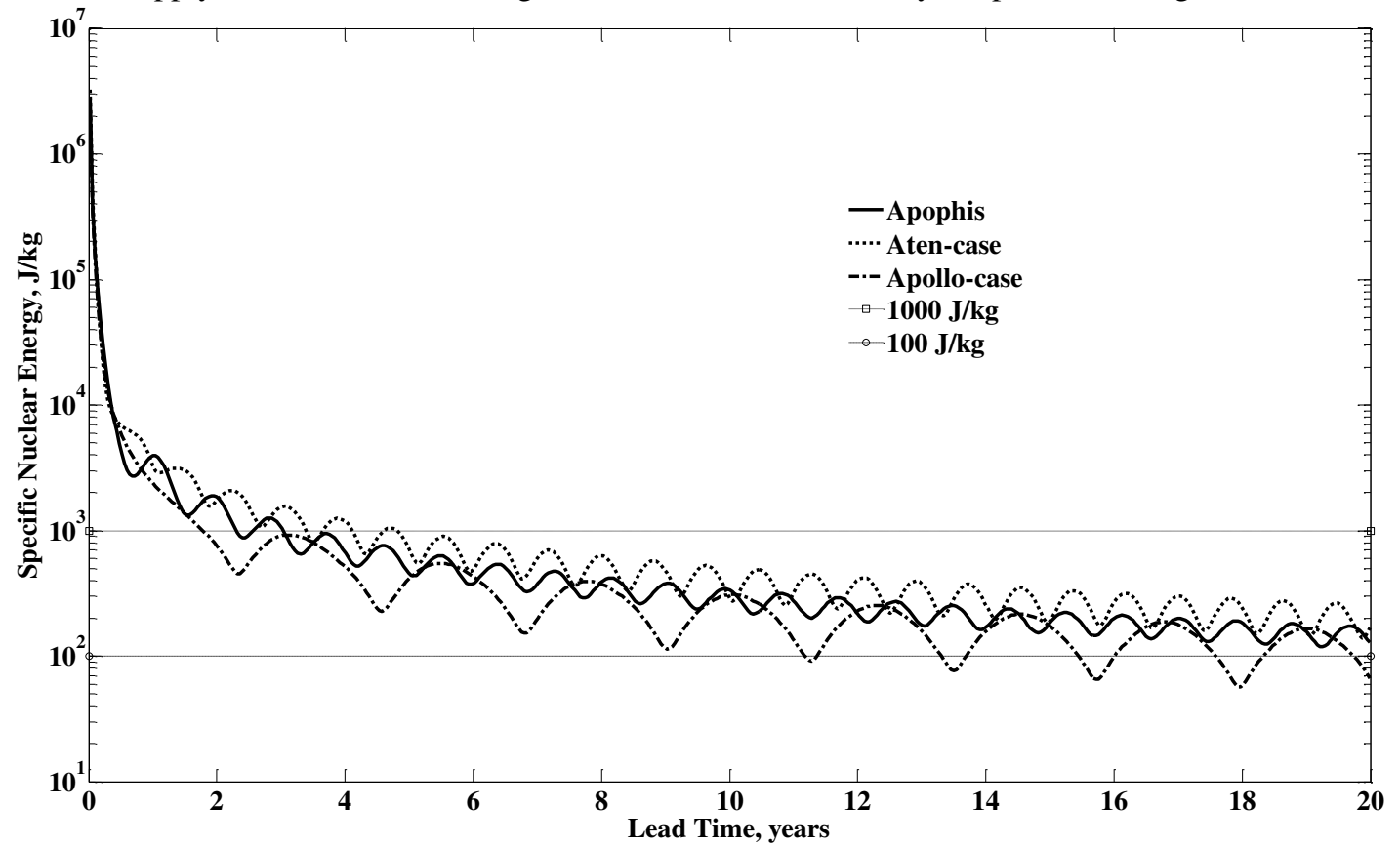

Fig. 4 Specific absorbed Nuclear Energy (SNE) provided for a nuclear interceptor ${ }^{[9]}$ attempting to deflect a threatening asteroid with a delta-velocity as specified in Fig. 2.

The two suggested limits $(1000 \mathrm{~J} / \mathrm{kg}$ and $100 \mathrm{~J} / \mathrm{kg}$ ) must be taken cautiously when assessing the likelihood of fragmentation triggered by a nuclear interceptor. Since these two limits were estimated from hypervelocity impact studies $^{[11]}$, the actual fragmentation energies for an asteroid being deflected by a nuclear device may be different, because of the different physical interaction. However, in this work it was considered that the shock wave caused by an impact and the thermal stress wave generated by the nuclear explosion are analogous, and therefore the associated fragmentation energies are expected to have similar orders of magnitude. It is also interesting to note that the SNE remains at higher levels of specific energy for long warning times, compared with the SKE. This is a consequence of the fact that at low levels of energies, an increasing percentage of the nuclear energy delivered is used to heat the asteroid up, without ablating material. There is therefore no change in linear momentum and the nuclear impactor becomes less efficient in terms of energy. Despite this loss of efficiency, the dry mass required for an equivalent deflection using a kinetic impactor will still be several orders of magnitude higher ${ }^{[9]}$.

\section{Statistical Model of a Fragmented Asteroid}

From the energetic requirements of a hazard mitigation mission, we can conclude that the possibility of an undesired break-up of an asteroid during a deflection attempt cannot be ignored. The consequences of an undesired fragmentation can be evaluated by studying the evolution of the cloud of fragments generated during the break-up process. The quantity, size and velocity of the pieces spawned by the fragmentation process needs then to be assessed. Building a deterministic dynamical model of the fragmentation and dispersion process is out of the scope of this work, instead the following sections propose a statistical model of the initial distribution of the fragments with associated positions and velocities and a methodology to calculate the probability to find the fragments in particular positions in space at different times.

\section{A. Fragmented Asteroid Dispersion}

The position and velocity of every piece of a fragmented asteroid can be described as a stochastic process, even if the dynamical system is deterministic, since the initial conditions of the system are not known and they can only be assessed through a probability density function. In particular, considering a scalar function describing the probability density of a dynamic system such as $\rho(\mathbf{X}(\mathrm{t}))=\rho(\mathbf{x}, \mathbf{v} ; \mathrm{t})$, where $\rho(\mathbf{x}, \mathbf{v} ; \mathrm{t})$ is the probability of a 
fragment to have position $\mathbf{X}$ and velocity $\mathbf{v}$ at a time t. The probability density function $\rho(\mathbf{X}(\mathrm{t}))$ relates to an initial probability density function $\rho(\mathbf{X}(0))$ through the equation:

$$
\rho(\mathbf{X}(\mathrm{t}))=\int_{\Gamma} \delta\left(\mathbf{X}(\mathrm{t})-\phi^{\mathrm{t}}(\mathbf{X}(0))\right) \rho(\mathbf{X}(0)) \mathrm{d} \Xi(0)
$$

where $\phi^{\mathrm{t}}(\mathbf{X}(0))$ denotes the flux of the system, or evolution of the state $\mathbf{X}(0)=[\mathbf{x}(0), \mathbf{v}(0)]^{\mathrm{T}}$ over a time-span $\mathrm{t}$ so that $\phi^{\mathrm{t}}(\mathbf{X}(0))$ is equal to $[\mathbf{x}(\mathrm{t}), \mathbf{v}(\mathrm{t})]^{\mathrm{T}}, \delta(\mathbf{y})$ is a multi-dimensional Dirac-delta, which represents the product of the one-dimensional Dirac-delta functions, that will allow a probability $\rho(\mathbf{X}(0))$ to be added to the total probability of $\rho(\mathbf{X}(\mathrm{t}))$, only if the initial state vector $\mathbf{X}(0)$ can effectively evolve to $\mathbf{X}(\mathrm{t})$, and finally, $\mathrm{d} \Xi(0)$ refers to the product of the one-dimensional differentials components of the vector $\mathbf{X}(0)$, i.e., $d x \cdot d y \cdot d z \cdot d v_{x} \cdot d v_{y} \cdot d v_{z}$, and defines the volume of an infinitesimal portion of the phase space $\Gamma$, which is the feasible phase space in which the system evolves.

If we introduce the new variable $\mathbf{z}=\phi^{\mathrm{t}}(\mathbf{X}(0))$ and the associated Jacobian determinant as $|\mathbf{J}|=\left|\frac{\partial \phi^{\mathrm{t}}(\mathbf{X}(0))}{\partial \mathbf{X}(0)}\right|$, it is possible to substitute the differential $\mathrm{d} \Xi(0)$ with $\mathrm{d} \zeta /\|\mathbf{J}\|$ in Eq.(5), where $\mathrm{d} \zeta$ is the product of the onedimensional differentials components of the vector $\mathbf{z}$ and $\|\mathbf{J}\|$ is the absolute value of the Jacobian determinant. This allows us to integrate Eq.(5) using the feasible phase space at time $t$ instead of the initial phase space, thus Eq.(5) results in the following integration:

$$
\rho(\mathbf{X}(\mathrm{t}))=\int_{\Gamma} \delta(\mathbf{X}(\mathrm{t})-\mathbf{z}) \rho\left(\phi^{-\mathrm{t}}(\mathbf{z}) ; 0\right) \frac{\mathrm{d} \zeta}{\|\mathbf{J}\|}
$$

Using the definition of a Dirac-delta function, the Eq.(6) resolves to:

$$
\rho(\mathbf{X}(\mathrm{t}))=\rho(\mathbf{x}, \mathbf{v} ; \mathrm{t})=\frac{1}{\|\mathbf{J}\|} \rho\left(\phi^{-\mathrm{t}}(\mathbf{x}, \mathbf{v}) ; 0\right)
$$

Eq.(7) tells us that the probability of a particular fragment having position $\mathbf{X}$ and velocity $\mathbf{V}$ at a time $t$ is the same probability of having the initial conditions that can make the fragment dynamically evolve to the particular state $\mathbf{X}(\mathrm{t})$ and then scaled by the absolute value of the Jacobian determinant $\|\mathbf{J}\|$. The Jacobian determinant $\|\mathbf{J}\|$ defines the evolution of the volume of the phase space from the initial time of the break-up to a given time t. By evoking Liouville's theorem, which states that for a Hamiltonian system the density of states in the phase space remains constant with time ${ }^{[27]}$, we know that $\|\mathbf{J}\|=1$, since the dynamical system used is a two body dynamics, thus Hamiltonian (i.e., the forces are velocity invariant) .

Transition Matrix

Eq.(5) can finally be expressed as:

$$
\rho(\mathbf{X}(\mathrm{t}))=\rho\left(\phi^{-\mathrm{t}}(\mathbf{x}, \mathbf{v}) ; 0\right)
$$

Hence, to compute the probability of having a fragment with a state vector $\mathbf{X}(\mathrm{t})$ it is necessary to calculate the predecessor of $\mathbf{X}(\mathrm{t})$ at break-up (i.e., $\mathbf{X}(0))$. A state transition matrix $\boldsymbol{\Phi}\left(\mathrm{t}, \mathrm{t}_{0}\right)$ such as:

$$
\boldsymbol{\Phi}\left(\mathrm{t}, \mathrm{t}_{0}\right)=\left[\begin{array}{ll}
\frac{\partial \mathbf{x}(\mathrm{t})}{\partial \mathbf{x}\left(\mathrm{t}_{0}\right)} & \frac{\partial \mathbf{x}(\mathrm{t})}{\partial \mathbf{v}\left(\mathrm{t}_{0}\right)} \\
\frac{\partial \mathbf{v}(\mathrm{t})}{\partial \mathbf{x}\left(\mathrm{t}_{0}\right)} & \frac{\partial \mathbf{v}(\mathrm{t})}{\partial \mathbf{v}\left(\mathrm{t}_{0}\right)}
\end{array}\right]
$$

will provide this direct mapping from an initial state vector $\mathbf{X}\left(\mathrm{t}_{0}\right)$ to the final state vector $\mathbf{X}(\mathrm{t})$ necessary to calculate Eq.(8).

$$
\left(\begin{array}{l}
\mathbf{x}(\mathrm{t}) \\
\mathbf{v}(\mathrm{t})
\end{array}\right)=\boldsymbol{\Phi}\left(\mathrm{t}, \mathrm{t}_{0}\right)\left(\begin{array}{l}
\mathbf{x}\left(\mathrm{t}_{0}\right) \\
\mathbf{v}\left(\mathrm{t}_{0}\right)
\end{array}\right)
$$


Since we are interested in studying the dispersion of a cloud of particles, we can work in relative coordinates to study the differences in position and velocity with respect to the unperturbed orbit of the asteroid prior to fragmentation. Eq.(10) can be simplified by assuming that all the fragmented particles depart from the centre of mass of the asteroid (i.e., the relative initial position $\Delta \mathbf{x}\left(\mathrm{t}_{0}\right)$ is 0 ), and by computing only the relative final position $\Delta \mathbf{x}(\mathrm{t})$ :

$$
\Delta \mathbf{x}(\mathrm{t})=\left[\frac{\partial \Delta \mathbf{x}(\mathrm{t})}{\partial \Delta \mathbf{v}\left(\mathrm{t}_{0}\right)}\right] \Delta \mathbf{v}\left(\mathrm{t}_{0}\right)
$$

This simplifies the problem considerably, since only the $3 \times 3$ relative transition matrix $\partial \Delta \mathbf{x}(\mathrm{t}) / \partial \Delta \mathbf{v}\left(\mathrm{t}_{0}\right)$ is required. The transition matrix $\partial \Delta \mathbf{x}(\mathrm{t}) / \partial \Delta \mathbf{v}\left(\mathrm{t}_{0}\right)$ is given by the product of the linear proximal motion equations and the Gauss' planetary equations (for further details see Vasile and Colombo ${ }^{[22]}$ ). This calculation provides a linear approximation of the nonlinear two body dynamics, but if the dispersive velocity is small compared to the nominal velocity of the unfragmented asteroid, it is an acceptable approximation.

Probability to find a particle in a certain position

Since we are interested in the probability of finding a fragment in a certain position in space at a particular time $\mathrm{t}$, the probability function $\rho(\mathbf{x}, \mathbf{v} ; \mathrm{t})$ will need to be integrated over all the feasible space of velocities:

$$
\mathrm{P}(\mathbf{x} ; \mathrm{t})=\int_{\Gamma} \rho(\mathbf{x}, \mathbf{v} ; \mathrm{t}) \mathrm{d} v(\mathrm{t})=\int_{\Gamma} \rho\left(\phi^{-\mathrm{t}}(\mathbf{x}, \mathbf{v}) ; 0\right) \mathrm{d} v(\mathrm{t})
$$

where $\mathrm{d} v(\mathrm{t})$ is the product of the one-dimensional differentials components of the velocity, $\mathrm{dv}_{\mathrm{x}} \cdot \mathrm{dv}_{\mathrm{y}} \cdot \mathrm{dv}_{\mathrm{z}}$.

Since the probability density function $\rho(\mathbf{x}, \mathbf{v} ; 0)$ is the probability to have a fragment in a position $\mathbf{x}(0)$ with velocity $\mathbf{v}(0)$ and having already assumed that the dispersion of fragments initiates from the centre of mass of the unfragmented asteroid, we can express $\rho(\mathbf{x}, \mathbf{v} ; 0)$ as the product of two separated probability density function:

$$
\rho(\mathbf{x}, \mathbf{v} ; 0)=\delta\left(\mathbf{x}(0)-\mathbf{r}_{\mathbf{0}}\right) \cdot \mathrm{G}(\mathbf{v}(0))
$$

where $\delta\left(\mathbf{x}(0)-\mathbf{r}_{\mathbf{0}}\right)$ is the probability of a particular fragment to have position $\mathbf{x}(0)-\mathbf{r}_{0}$, where $\mathbf{r}_{0}$ is the position of the centre of mass of the unfragmented asteroid at $t=0$, and $\mathrm{G}(\mathbf{v}(0))$ is the probability that the same fragment has velocity $\mathbf{v}(0)$. Now, Eq.(12) can be rewritten using Eq.(13) as:

$$
\mathrm{P}(\mathbf{x} ; \mathrm{t})=\int_{\Gamma} \delta\left(\phi^{-\mathrm{t}}(\mathbf{x}, \mathbf{v})_{\mathbf{r}}-\mathbf{r}_{\mathbf{0}}\right) \cdot \mathrm{G}\left(\phi^{-\mathrm{t}}(\mathbf{x}, \mathbf{v})_{\mathbf{v}}\right) \mathrm{d} v(\mathrm{t})
$$

where $\phi^{-t}(\mathbf{x}, \mathbf{v})_{\mathbf{r}}$ and $\phi^{-t}(\mathbf{x}, \mathbf{v})_{\mathbf{v}}$ are, respectively, the components of the position and velocity of the flux $\phi^{-t}(\mathbf{x}, \mathbf{v})$. Now, similarly to what was done with Eq.(5), the element of volume of the space of velocities $\mathrm{d} v(\mathrm{t})$ can be related to the element $d \xi(0)=d x \cdot d y \cdot d z$ through their Jacobian:

$$
\mathrm{d} v(\mathrm{t})=\left\|\frac{\partial \mathbf{v}(\mathrm{t})}{\partial \mathbf{x}(0)}\right\| \mathrm{d} \xi(0)
$$

allowing us to solve the integral in Eq.(12) as:

$$
\mathrm{P}(\mathbf{x} ; \mathrm{t})=\left\|\frac{\partial \mathbf{v}(\mathrm{t})}{\partial \mathbf{x}(0)}\right\| \mathrm{G}\left(\phi^{-\mathrm{t}}\left(\mathbf{x}, \mathbf{v}_{*}\right)_{\mathbf{v}}\right)
$$

where $\mathbf{v}_{*}$ is the solution of the equation:

$$
\phi^{-\mathrm{t}}\left(\mathbf{x}, \mathbf{v}_{*}\right)_{\mathbf{r}}=\mathbf{r}_{\mathbf{0}}
$$

so that $\delta\left(\phi^{-\mathrm{t}}(\mathbf{x}, \mathbf{v})_{\mathbf{r}}-\mathbf{r}_{\mathbf{0}}\right)$ is 1 . Besides, the absolute value of the Jacobian in Eq.(16) can be written as:

$$
\left\|\frac{\partial \mathbf{v}(\mathrm{t})}{\partial \mathbf{x}(0)}\right\|=\frac{1}{\left\|\frac{\partial \mathbf{x}(0)}{\partial \mathbf{v}(\mathrm{t})}\right\|}
$$


so that the absolute value of the determinant $\|\partial \Delta \mathbf{x}(0) / \partial \Delta \mathbf{v}(\mathrm{t})\|$ can be calculated by using the state transition matrix $\partial \Delta \mathbf{x} / \partial \Delta \mathbf{v}$ in Eq.(11) only by substituting the corresponding times t.

Finally, the probability to find a piece of asteroid in a particular position relative to the unperturbed orbit at a given time after a fragmentation is given by:

$$
\mathrm{P}(\Delta \mathbf{x} ; \mathrm{t})=\frac{1}{\left\|\frac{\partial \Delta \mathbf{x}\left(\mathrm{t}_{0}\right) \|}{\partial \Delta \mathbf{v}(\mathrm{t})}\right\|} \mathrm{G}\left(\left[\frac{\partial \Delta \mathbf{x}(\mathrm{t})}{\partial \Delta \mathbf{v}\left(\mathrm{t}_{0}\right)}\right]^{-1} \Delta \mathbf{x}(\mathrm{t})\right)
$$

Note that, the proposed model assumes implicitly that the asteroid fragments in such a way that the centre of mass of the large fragments is initially close to the core of the asteroid. In a future work, we will analyze the case in which this hypothesis does not apply.

\section{B. Velocity Dispersion Model}

The probability density function defined in Eq.(13) depends on two terms, a Dirac delta such as $\delta\left(\mathbf{x}(0)-\mathbf{r}_{0}\right)$ for the position, which is equivalent to one Dirac delta function for each one of the components of the vector $\mathbf{x}(0)$, and a function $G(\mathbf{v}(0))$ that describes the dispersion of the values of the initial velocity $\mathbf{v}(0)$. For the latter purpose, we will use three Gaussian distribution; each Gaussian distribution will describe the velocity dispersion in one direction of the cartesian $\hat{\mathrm{t}}-\hat{\mathrm{n}}-\hat{\mathrm{h}}$ reference frame or tangential, normal and out-of-plane direction:

$$
\mathrm{G}\left(\mathrm{v}_{\mathrm{t}}(0), \mathrm{v}_{\mathrm{n}}(0), \mathrm{v}_{\mathrm{h}}(0)\right)=\frac{1}{\sigma_{\mathrm{t}} \sqrt{2 \pi}} \mathrm{e}^{\frac{-\left(\mathrm{v}_{\mathrm{t}}-\mu_{\mathrm{t}}\right)^{2}}{2 \sigma_{\mathrm{t}}^{2}}} \cdot \frac{1}{\sigma_{\mathrm{n}} \sqrt{2 \pi}} \mathrm{e}^{\frac{-\left(\mathrm{v}_{\mathrm{n}}-\mu_{\mathrm{n}}\right)^{2}}{2 \sigma_{\mathrm{n}}^{2}}} \cdot \frac{1}{\sigma_{\mathrm{h}} \sqrt{2 \pi}} \mathrm{e}^{\frac{-\left(\mathrm{v}_{\mathrm{h}}-\mu_{\mathrm{h}}\right)^{2}}{2 \sigma_{\mathrm{h}}^{2}}}
$$

Six parameters will be needed in order to define the dispersion of velocities: three mean velocities $\boldsymbol{\mu}=\left[\begin{array}{lll}\mu_{\mathrm{t}} & \mu_{\mathrm{n}} & \mu_{\mathrm{h}}\end{array}\right]$, and three standard deviations $\boldsymbol{\sigma}=\left[\begin{array}{lll}\sigma_{\mathrm{t}} & \sigma_{\mathrm{n}} & \sigma_{\mathrm{h}}\end{array}\right]$.

Assuming a kinetic impactor scenario, we can hypothesize that an infinitesimal instant after the impact, but before the fragmentation takes place, the system asteroid-spacecraft form a single object, which moves according to the law of conservation of linear momentum. In fact, after the kinetic impactor mission triggers a catastrophic fragmentation, it is reasonable to think that the system asteroid-spacecraft would preserve the total linear momentum. Hence, given the SKE of a particular collision, Eq.(4) will provide the change of velocity of the centre of mass of the system only by considering the momentum enhancement factor $\beta$ equal to 1 . It also seems sensible to think of the mean vector $\boldsymbol{\mu}=\left[\begin{array}{lll}\mu_{\mathrm{t}} & \mu_{\mathrm{n}} & \mu_{\mathrm{h}}\end{array}\right]$ as the change of velocity of the centre of mass, since the highest probability to find a fragment should be at the centre of mass of the system. As a result, the norm of the mean of the dispersion should be:

$$
|\boldsymbol{\mu}|=\left|\Delta \mathbf{v}_{\mathrm{a}}\right|=\frac{\sqrt{2 \mathrm{M}_{\mathrm{a}} \mathrm{m}_{\mathrm{s} / \mathrm{c}} \mathrm{SKE}}}{\left(\mathrm{M}_{\mathrm{a}}+\mathrm{m}_{\mathrm{s} / \mathrm{c}}\right)}
$$

The direction of $|\boldsymbol{\mu}|$ is defined by the direction of the impact relative velocity $\Delta \mathbf{v}_{\mathrm{s} / \mathrm{c}}$. Since the trajectory of a kinetic impactor should be designed to achieve the maximum possible deviation, $|\boldsymbol{\mu}|$ should be directed along the tangential direction ${ }^{[22]}$. Accordingly, given the SKE of the collision, the mean velocity dispersion vector can be taken as:

$$
\boldsymbol{\mu}=\left[\begin{array}{ccc}
\frac{\sqrt{2 \mathrm{M}_{\mathrm{a}} \mathrm{m}_{\mathrm{s} / \mathrm{c}} \mathrm{SKE}}}{\left(\mathrm{M}_{\mathrm{a}}+\mathrm{m}_{\mathrm{s} / \mathrm{c}}\right)} & 0 & 0
\end{array}\right]
$$

Just as it is sensible to think that after a dish has shattered on the floor, the smallest fragments are generally found the furthest, one would expect that the smaller the fragments of the asteroid are the larger their velocity dispersion $\boldsymbol{\sigma}=\left[\begin{array}{lll}\sigma_{\mathrm{t}} & \sigma_{\mathrm{n}} & \sigma_{\mathrm{h}}\end{array}\right]$ will be. The mass of the fragment must therefore have an influence on the dispersion of velocities. Let us assume that a fragment with mass $m_{\mathrm{i}}$ has a velocity $\Delta \mathrm{v}_{\mathrm{i}}$ defined by an inelastic collision such 
that (note that, in the following, in order to simplify the equations, it is considered that $\mathrm{m}_{\mathrm{s} / \mathrm{c}}$ is always orders of magnitude smaller than both $\mathrm{M}_{\mathrm{a}}$ and $\mathrm{m}_{\mathrm{i}}$, thus $\mathrm{M}_{\mathrm{a}}+\mathrm{m}_{\mathrm{s} / \mathrm{c}} \approx \mathrm{M}_{\mathrm{a}}$ and $\mathrm{m}_{\mathrm{i}}+\mathrm{m}_{\mathrm{s} / \mathrm{c}} \approx \mathrm{m}_{\mathrm{i}}$ ):

$$
\mathrm{m}_{\mathrm{i}} \Delta \mathrm{v}_{\mathrm{i}} \approx \mathrm{m}_{\mathrm{s} / \mathrm{c}} \Delta \mathrm{v}_{\mathrm{SKE} \cdot \mathrm{m}_{\mathrm{i}}}
$$

where $\Delta \mathrm{v}_{\text {SKE } \cdot \mathrm{m}_{\mathrm{i}}}$ comes from the fraction of collisional energy SKE that fragment $\mathrm{m}_{\mathrm{i}}$ absorbs and is defined as:

$$
\Delta \mathrm{v}_{\mathrm{SKE} \cdot \mathrm{m}_{1}}=\sqrt{\frac{2 \cdot \mathrm{SKE} \cdot \mathrm{m}_{1}}{\mathrm{~m}_{\mathrm{s} / \mathrm{c}}}}
$$

On the other hand, according to Section III.B (see Eq.(4)), the real impact occurs between the unfragmented asteroid with mass $M_{a}$ and the spacecraft with mass $m_{s / c}$ at a relative velocity of:

$$
\Delta \mathrm{v}_{\mathrm{s} / \mathrm{c}}=\sqrt{\left(2 \cdot \mathrm{SKE} \cdot \mathrm{M}_{\mathrm{a}}\right) / \mathrm{m}_{\mathrm{s} / \mathrm{c}}}
$$

Writing Eq.(24) as a function of the real impact velocity $\Delta \mathrm{v}_{\mathrm{s} / \mathrm{c}}$ of the spacecraft leads us to:

$$
\Delta \mathrm{v}_{\mathrm{SKE} \cdot \mathrm{m}_{1}}=\sqrt{\frac{\mathrm{m}_{1}}{\mathrm{M}_{\mathrm{a}}}} \cdot \Delta \mathrm{v}_{\mathrm{s} / \mathrm{c}}
$$

Using the virtual inelastic collision Eq.(23) and Eq.(26), we can write $\Delta \mathrm{v}_{\mathrm{i}}$ as:

$$
\Delta \mathrm{v}_{\mathrm{i}}=\frac{\mathrm{m}_{\mathrm{s} / \mathrm{c}}}{\mathrm{m}_{\mathrm{i}}} \sqrt{\frac{\mathrm{m}_{\mathrm{i}}}{\mathrm{M}_{\mathrm{a}}}} \cdot \Delta \mathrm{v}_{\mathrm{s} / \mathrm{c}}
$$

As it was said before, the centre of mass of the cloud of fragments is likely to follow the law of conservation of linear momentum (i.e., $\mathrm{M}_{\mathrm{a}} \Delta \mathrm{v}_{\mathrm{a}} \approx \mathrm{m}_{\mathrm{s} / \mathrm{c}} \Delta \mathrm{v}_{\mathrm{s} / \mathrm{c}}$ ), hence Eq.(27) finally settles down to the following expression:

$$
\Delta \mathrm{v}_{\mathrm{i}}=\sqrt{\frac{\mathrm{M}_{\mathrm{a}}}{\mathrm{m}_{\mathrm{i}}}} \cdot \Delta \mathrm{v}_{\mathrm{a}}
$$

Note that Eq. (28) is denoting a power law relationship between the mass of the different fragments and their velocity as:

$$
\frac{1}{2} \mathrm{~m} \Delta \mathrm{v}^{\mathrm{x}}=\text { constant }
$$

where the exponent $\mathrm{x}$ is equal 2. Hence, we are assuming a homogenous distribution of the translational kinetic energy among all the fragments, or equipartition of translational kinetic energy. Wiesel ${ }^{[14]}$ also suggest an equipartition effect when studying the explosion of objects such as spacecraft in Earth orbit. Figure 7 in Davis and Ryan $^{[11]}$ shows two different experiments on collisional disruption with an accumulative ejecta mass at velocities greater than a given velocity $\mathrm{v}$ with slopes of -1.92 and -1.41 . By using a power law distribution of fragments as described in Greenberg et al. ${ }^{[12]}$ (or later in this paper) a relation as Eq.(29) can be extrapolated, which yields exponents x equal to 2.74 and 1.58 respectively. Also Gault et al. ${ }^{[28]}$ found an "accumulative" mass-velocity relation with an slope of -2.25 for his cratering experiments, which considering a power law distribution of fragments also accounts for quasi-homogeneous distribution of translational kinetic energy. On the other hand, results from Nakamura et al. ${ }^{[29]}$ show a higher x exponent, between 3 and 6, which would result in lower fragment velocity.

Recalling the definition of standard deviation, $\sigma=\sqrt{\left\langle\Delta \mathbf{v}^{2}\right\rangle-\langle\Delta \mathbf{v}\rangle^{2}}$, where $\langle x\rangle$ denotes the average of all elements $\mathrm{x}$, and assuming that $\langle\boldsymbol{\Delta} \mathbf{v}\rangle$ is equal to zero for a homogeneous spherical dispersion from the centre of mass of the cloud of fragments, we can compute the norm of the standard deviation of velocity $\sigma\left(\mathrm{m}_{1}\right)$ using Eq.(28) as:

$$
\sigma\left(\mathrm{m}_{\mathrm{l}}\right)=\sqrt{\frac{\mathrm{M}_{\mathrm{a}}}{\mathrm{m}_{\mathrm{1}}}} \cdot \sigma_{0}
$$

where $\sigma_{0}$ is defined as: 


$$
\sigma_{0}=\frac{\Delta \mathrm{v}_{\mathrm{a}}}{\mathrm{k}}
$$

with $\mathrm{k}$ a constant value. The constant $\mathrm{k}$ is 1 if we consider the velocity of the fragment with mass $\mathrm{m}_{\mathrm{i}}$ as in Eq.(28).

In fact, one could think of $\mathrm{k}$ as the efficiency of transmission of the collisional energy. If part of the collisional energy is lost in processes such as melting or breaking, one could expect $\mathrm{k}$ to be larger than 1 , on the other hand, $\mathrm{k}$ could also be smaller than 1 for fragments coming from areas in the asteroid where there was a higher reservoir of collisional energy, e.g., close to the impact site. Therefore, it would be sensible to expect that small fragments may have k equal to 1 or smaller, since small fragments must come from areas with higher reservoir of collisional energy so that this energy was able to break the material to smaller sizes. Large fragments may have instead $\mathrm{k}$ larger than 1 from opposite reasons. Using experimental data published by Davis and Ryan ${ }^{[11]}$, one can fit their experiments with available velocity dispersion data to find an average value of $\mathrm{k}$. By doing so, $\mathrm{k}$ results in 1.4, thus,

$$
\sigma_{0}=\frac{\Delta \mathrm{v}_{\mathrm{a}}}{1.4}
$$

To finish, the norm of standard deviation of velocity is $\sigma\left(\mathrm{m}_{1}\right)$ as in Eq.(30), and since we assume a homogeneous spherical dispersion on the initial velocities at the break-up point, we can write the vector of the standard deviation as assuming three equal 1-dimensional values:

$$
\boldsymbol{\sigma}=\left[\frac{1}{\sqrt{3}} \sqrt{\frac{\mathrm{M}_{\mathrm{a}}}{\mathrm{m}_{\mathrm{l}}}} \cdot \sigma_{0} \frac{1}{\sqrt{3}} \sqrt{\frac{\mathrm{M}_{\mathrm{a}}}{\mathrm{m}_{\mathrm{i}}}} \cdot \sigma_{0} \frac{1}{\sqrt{3}} \sqrt{\frac{\mathrm{M}_{\mathrm{a}}}{\mathrm{m}_{\mathrm{1}}}} \cdot \sigma_{0}\right]
$$

\section{On the Nature of the Cloud of Fragments}

This section will give some insight into the nature of the cloud of fragments formed after the break-up. As previous work on the dispersion of non-interacting particles has already shown ${ }^{[30]}$, the apparent shape of the cloud of fragments (or the probability density function of the particles in the cloud) will evolve as a pulsing ellipsoid with a semimajor axis lengthening with time. The apparent pulsing of this ellipsoid is due to both the collapse of the out-ofplane component of the dispersion, which happens twice per orbit, and the collapse of the semiminor axis of the ellipsoid, which occurs when the tangential and the normal component of the dispersion align, happening also twice per orbit. This, of course, would summarize the apparent evolution of a fragmented asteroid only if its fragments are small enough and move quick enough to have a negligible gravitational interaction. In order to assess, then, the magnitude of the gravitational interactions, we will need first to have some insight into the statistical composition of the cloud of fragments in terms of quantity and size of its particles.

\section{A. Fragment Size Distribution}

It is out of the scope of this paper to describe the physics of the fragmentation of a brittle solid, such as an asteroid, and a simple statistical distribution of fragments will serve better to our purposes, which are to discern the intrinsic risks of the asteroid hazard mitigation. The aim here is only to discern the intrinsic risks of an impulsive asteroid hazard mitigation strategy. This purpose can be achieved by estimating the approximate number of fragments within a given range of mass. With this, we will also be able to perform a preliminary analysis of the gravitational interaction among the different components of the cloud.

Early works in collisional fragmentation have used accumulative power law distribution to model fragment size distribution $^{[12]}$. Two- or three- segments power laws have been found to fit much better to experimental data ${ }^{[11 ; 31]}$, particularly when the fragmentation data comprises sizes many orders of magnitude smaller than the original size. However, for the analysis carried out here we will use only one segment accumulative power law distribution such as:

$$
\mathrm{N}(\geq \mathrm{m})=\mathrm{Cm}^{-\mathrm{b}}
$$

since this is already an acceptable approximation for a qualitative analysis inside a range of 3 orders of magnitude of the fragment mass. Eq.(34) provides the number of fragments above a given mass $\mathrm{m}$, therefore if $\mathrm{m}_{\max }$ is the mass of the largest fragment, $\mathrm{N}\left(\geq \mathrm{m}_{\max }\right)$ must be 1 , and thus the constant $\mathrm{C}$ must be:

$$
\mathrm{C}=\mathrm{m}_{\max } \mathrm{b}
$$

Now, If we integrate the mass over all possible fragment sizes, the total mass must be equal to the unfragmented asteroid mass $\mathrm{M}_{\mathrm{a}}$ : 


$$
\mathrm{M}_{\mathrm{a}}=\int_{1}^{\infty} \mathrm{m} \cdot \mathrm{dN}(>\mathrm{m})=\left[\frac{\mathrm{bC}}{(1-\mathrm{b})}\right] \mathrm{m}_{\max }^{1-\mathrm{b}}
$$

Using Eq.(35) in Eq.(36), the exponent $b$ becomes a function only of the ratio between the largest fragment mass $\mathrm{m}_{\max }$ and the total mass of the asteroid $\mathrm{M}_{\mathrm{a}}$ :

$$
\mathrm{b}=\left(1+\frac{\mathrm{m}_{\mathrm{max}}}{\mathrm{M}_{\mathrm{a}}}\right)^{-1}
$$

where the fraction $m_{\text {max }} / M_{a}$ is the fragmentation ratio $f_{r}$.

Fig. 5 shows the number of fragments that a one-segment power law distribution such as Eq.(34) predicts for three different catastrophic fragmentations of Apophis test case: $f_{r}=0.5$ (black bars), $f_{r}=0.25$ (white bars) and $\mathrm{f}_{\mathrm{r}}=0.1$ (grey bars). Only the range of fragments that can pose threat to Earth are shown in the figure, thus from $\mathrm{m}_{\max }$ to, roughly, the mass of a $40 \mathrm{~m}$ diameter rocky object. The number of fragments are counted inside equally wide and equispaced boxes, the width of the plotted bar is only chosen for clarity.

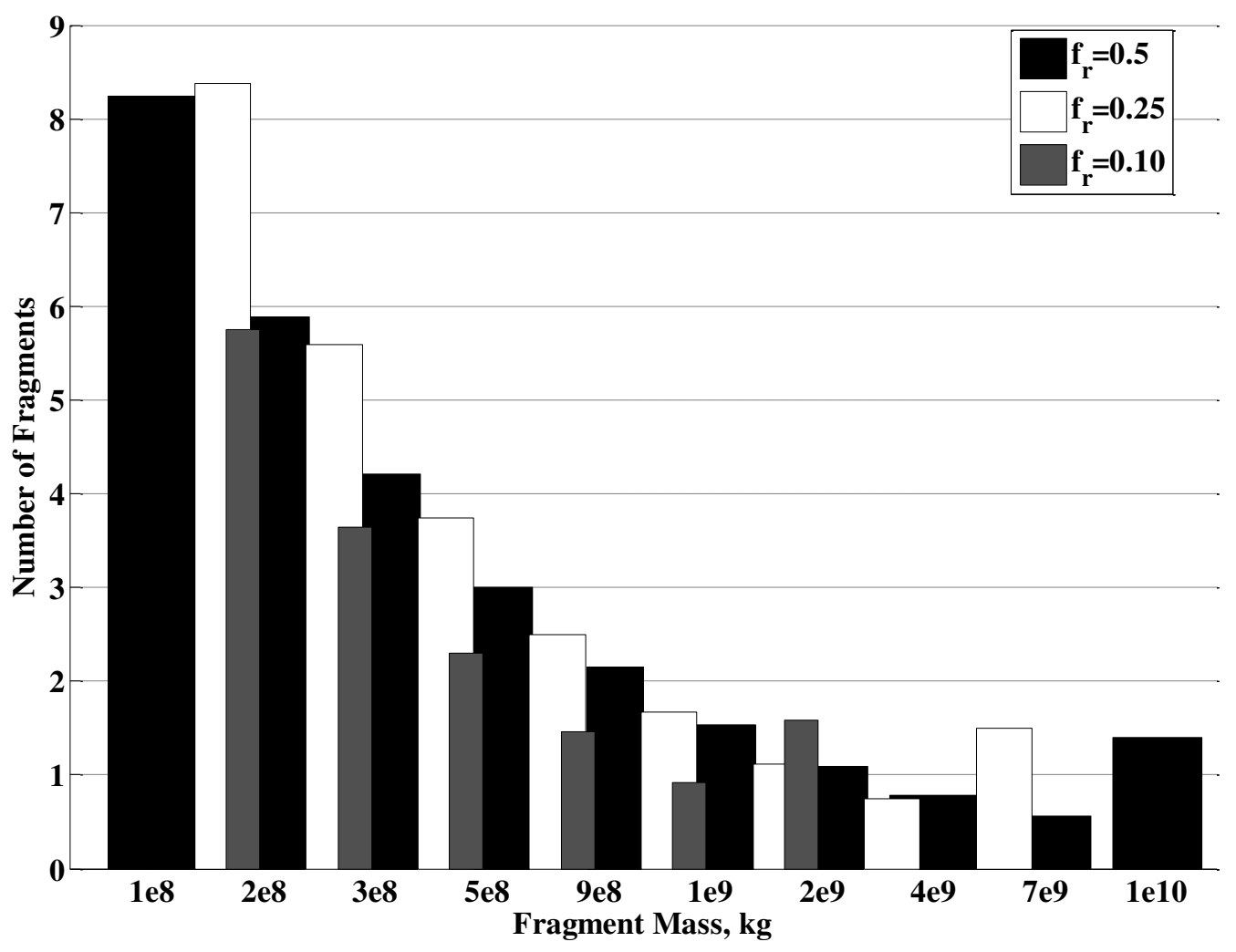

Fig. 5 Approximated number of pieces expected to be found in a fragmentation cloud of an asteroid with $2.7 \times 10^{10} \mathrm{~kg}$ of mass resulting from disruptions with $\mathrm{f}_{\mathrm{r}}=0.5$ (black bars), $\mathrm{f}_{\mathrm{r}}=0.25$ (white bars) and $\mathrm{f}_{\mathrm{r}}=0.1$ (grey bars). The largest fragment, i.e., surviving mass of the asteroid, is counted in the initial bin of the histogram for each level of disruption.

It is interesting to note that the higher the level of disruption the lesser the number of dangerous fragments. In fact, the total mass of the dangerous fragments is $85 \%, 63 \%$ and $30 \%$ the mass of the unshattered asteroid for fragmentation ratios $\mathrm{f}_{\mathrm{r}}$ of $0.5,0.25$ and 0.1 respectively, thus, a priori, the higher the level of fragmentation the lesser the risk for the Earth. By definition, a fragmentation equal to the critical specific energy $Q *$ triggers a barely 
catastrophical fragmentation, $\mathrm{f}_{\mathrm{r}}=0.5$, yet if the energy provided by a impulsive mitigation mission differs from this, a different level of disruption should be expected. In fact, the fragmentation ratio $f_{r}$ in Benz and Asphaug ${ }^{[32]}$ simulations adjusted very well to a simple linear function of the ratio between the specific energy applied and the critical specific energy Q*, at least for a range between 0.5 to 2 times $Q^{*}$. Although Benz and Asphaug ${ }^{[32]}$, s results cannot be extrapolated to this work since their simulations considered only impact velocities up to $5 \mathrm{~km} / \mathrm{s}$, they emphasise that for specific energies only a few times larger than $\mathrm{Q}^{*}$, the fragmentation ratio could decrease even more than one order of magnitude. In all the remaining analysis, we will consider three different fragmentation ratios $\left(f_{r}=0.5, f_{r}=0.25\right.$ and $\left.f_{r}=0.1\right)$ in order to represent different possible break-up scenarios.

\section{B. Gravity Re-aggregation}

Both the unshattered asteroids used in this paper or the largest fragment resultant from their catastrophic fragmentation have very weak gravitational attraction due to their small mass. Despite this, their gravity will still be the principal source of gravitational force inside a sphere centered on these bodies and smaller than a kilometer radius. Although this is a very small volume of interplanetary space, the gravitational interaction among the different fragments, during the initial moments after the break-up, may play a very important role on the nature of the dispersive cloud of fragments. It may well happen that some of the fragments, most probably the larger ones, are provided with very little relative velocity with respect to the largest fragments of the cloud, and so, they may spend too much time in very close proximity with them. Those fragments will not disperse as the dispersion model foresees, but rather, will re-accumulate into rubble piles or become orbiting satellites of a larger fragment ${ }^{[33 ; 34]}$.

Considering the coarse statistical description of the model, it is deemed that a full treatment of the gravity perturbation on each fragment due to the gravitational attraction of all the other fragments is not necessary at this stage, nevertheless the total re-accumulated mass needs to be determined. Hence, as a first approximation, we will compute the percentage of mass (or number of fragments) that are able to escape from the vicinity of the largest and most massive fragment, avoiding being either re-accumulated or becoming an orbiting satellite. The final population of fragments and the mass of the largest one will be then updated.

If the escape velocity of a fragment $f_{i}$ is computed as:

$$
\mathrm{v}_{\mathrm{esc}}=\sqrt{\frac{2 \mathrm{Gm}_{\mathrm{max}}}{\mathrm{r}_{\mathrm{i}}}}
$$

where $G$ is the Newtonian constant of gravitation and $r_{i}$ is the minimum distance between the centers of two spherical volumes with mass $m_{\max }$ and $m_{i}$ and density $2600 \mathrm{~kg} / \mathrm{m}^{3}$, the percentage of fragments $f_{i}$ avoiding being captured by the gravity pull of the largest fragment $f_{\max }$ can be calculated by computing the probability of those fragments to have a relative velocity with respect to the largest fragment $f_{\max }$ higher than the escape velocity in Eq.(38). Thus, the probability that a fragment $f_{i}$ escapes from the gravity of $f_{\max }$ should be computed by integrating the following expression:

$$
\mathrm{P}\left(\Delta \mathrm{v}>\mathrm{v}_{\mathrm{esc}}\right)=\int_{-\infty}^{\infty} \int_{-\infty}^{\infty} \int_{-\infty}^{\infty} \int_{-\infty}^{\infty} \int_{-\infty}^{\infty} \int_{-\infty}^{\infty} \mathrm{F}\left(\mathbf{v}_{\mathrm{f}_{\mathrm{i}}}(0), \mathbf{v}_{\max }(0)\right) \cdot \mathrm{d} v_{\mathrm{f}_{\mathrm{i}}}(0) \mathrm{d} v_{\max }(0)
$$

where $\mathrm{d} v_{\mathrm{f}_{\mathrm{i}}}(0)=\mathrm{dv}_{\mathrm{tf}_{\mathrm{i}}} \mathrm{dv}_{\mathrm{nf}_{\mathrm{i}}} \mathrm{dv}_{\mathrm{hf}_{\mathrm{i}}}, \mathrm{d} v_{\max }(0)=\mathrm{dv}_{\mathrm{t}_{\max }} \mathrm{dv}_{\mathrm{n}_{\max }} \mathrm{dv}_{\mathrm{h}_{\text {max }}}$ and the function $\mathrm{F}$ is:

$$
\begin{aligned}
& \mathrm{F}\left(\mathbf{v}_{\mathrm{f}_{\mathrm{i}}}(0), \mathrm{v}_{\text {max }}(0)\right)= \\
& \left\{\begin{array}{l}
\mathrm{G}\left(\mathrm{v}_{\mathrm{t}_{\mathrm{i}}}(0), \mathrm{v}_{\mathrm{nf}_{\mathrm{i}}}(0), \mathrm{v}_{\mathrm{h} \mathrm{f}_{\mathrm{i}}}(0)\right) \cdot \mathrm{G}\left(\mathrm{v}_{\mathrm{t}_{\text {max }}}(0), \mathrm{v}_{\mathrm{n}_{\text {max }}}(0), \mathrm{v}_{\mathrm{h}_{\text {max }}}(0)\right),\left|\mathbf{v}_{\mathrm{f}_{\mathrm{i}}}(0)-\mathbf{v}_{\text {max }}(0)\right|>\mathrm{v}_{\text {esc }} \\
0, \text { otherwise }
\end{array}\right.
\end{aligned}
$$

Integral Eq.(39) is computationally very expensive, thus a method to reduce the computational time without impairing the result of the integration was needed. Using the law of cosines, we can relate the modulus of the relative velocity $\mathbf{v}_{\mathrm{f}_{\mathrm{i}}}(0)-\mathbf{v}_{\max }(0)$ with the norm of the velocity $\mathbf{v}_{\mathrm{f}_{\mathrm{i}}}(0)$ and $\mathbf{v}_{\max }(0)$ as:

$$
\left|\mathbf{v}_{\mathrm{f}_{\mathrm{i}}}(0)-\mathbf{v}_{\max }(0)\right|^{2}=\left|\mathbf{v}_{\mathrm{f}_{\mathrm{i}}}(0)\right|^{2}+\left|\mathbf{v}_{\max }(0)\right|^{2}-2 \cdot\left|\mathbf{v}_{\mathrm{f}_{\mathrm{i}}}(0)\right| \cdot\left|\mathbf{v}_{\max }(0)\right| \cdot \cos (\gamma)
$$


where $\gamma$ is the angle between the two vectors $\mathbf{v}_{\mathrm{f}_{\mathrm{i}}}(0)$ and $\mathbf{v}_{\max }(0)$. Since the velocity distribution accounts for three equally distributed cartesian components, that is, equal mean and standard deviation for all components, angle $\gamma$ has a homogeneous probability to have a value between 0 and 360 degrees. Then, by averaging $\cos (\gamma)$ in Eq.(41) between 0 and 360 degrees, resulting in $\langle\cos (\gamma)\rangle=0$, we obtain an average modulus of the relative velocity as:

$$
\left|\mathbf{v}_{\mathrm{f}_{\mathrm{i}}}(0)-\mathbf{v}_{\max }(0)\right|^{2}=\left|\mathbf{v}_{\mathrm{f}_{\mathrm{i}}}(0)\right|^{2}+\left|\mathbf{v}_{\max }(0)\right|^{2}
$$

Using Eq.(42), the integral Eq.(39) can be reduced to a double integral if we use the norms of the vectors $\mathbf{v}_{\mathrm{f}_{\mathrm{i}}}(0)$ and $\mathbf{v}_{\text {max }}(0)$ instead of the six cartesian components:

$$
\mathrm{P}\left(\Delta \mathrm{v}>\mathrm{v}_{\mathrm{esc}}\right)=\int_{-\infty}^{\infty} \int_{-\infty}^{\infty} \mathrm{l}\left(\mathrm{v}_{\mathrm{f}_{\mathrm{i}}}(0), \mathrm{v}_{\max }(0)\right) \cdot \mathrm{dv}_{\mathrm{f}_{\mathrm{i}}}(0) d \mathrm{v}_{\max }(0)
$$

where function 1 becomes:

$$
\begin{aligned}
& 1\left(\mathrm{v}_{\mathrm{f}_{\mathrm{i}}}(0), \mathrm{v}_{\max }(0)\right)= \\
& \left\{\begin{array}{l}
\frac{1}{\sigma_{\mathrm{f}_{\mathrm{i}}} \sqrt{2 \pi}} \mathrm{e}^{\frac{-\mathrm{v}_{\mathrm{f}_{\mathrm{i}}}^{2}}{2 \sigma_{\mathrm{f}_{\mathrm{i}}}^{2}}} \cdot \frac{1}{\sigma_{\max } \sqrt{2 \pi}} \mathrm{e}^{\frac{-\mathrm{v}_{\max } 2^{2}}{2 \sigma_{\max }{ }^{2}}}, \sqrt{\left|\mathbf{v}_{\mathrm{f}_{\mathrm{i}}}(0)\right|^{2}+\left|\mathbf{v}_{\max }(0)\right|^{2}}>\mathrm{v}_{\text {esc }} \\
0, \quad \text { otherwise }
\end{array}\right.
\end{aligned}
$$

$$
\text { with } \sigma_{\mathrm{f}_{\mathrm{i}}}=\sqrt{\frac{\mathrm{M}_{\mathrm{a}}}{\mathrm{m}_{\mathrm{i}}}} \cdot \sigma_{0} ; \sigma_{\max }=\sqrt{\frac{\mathrm{M}_{\mathrm{a}}}{\mathrm{m}_{\max }}} \cdot \sigma_{0}
$$

Expression Eq.(44) does not include the mean of the distribution, since both fragments have, by definition, the same mean velocity.

Fig. 6 and Fig. 7 show the results of the integral Eq.(43) on the fragmentation of the asteroids Apophis, Atencase and Apollo-case. An interesting conclusion highlighted by the two figures concerns rubble pile asteroids; 100 $\mathrm{J} / \mathrm{kg}$ of impact energy may be the minimum energy required to fragment an asteroid, only if the asteroid happen to have a rubble-pile structure, if the fragmentation occurs at this level of energy, the velocities provided to the different spawned fragments would certainly not be enough to completely disperse the asteroid, which, most likely, would become a rubble pile asteroid again, losing only a small fraction of its mass during the process of break-up and subsequent re-aggregation. 


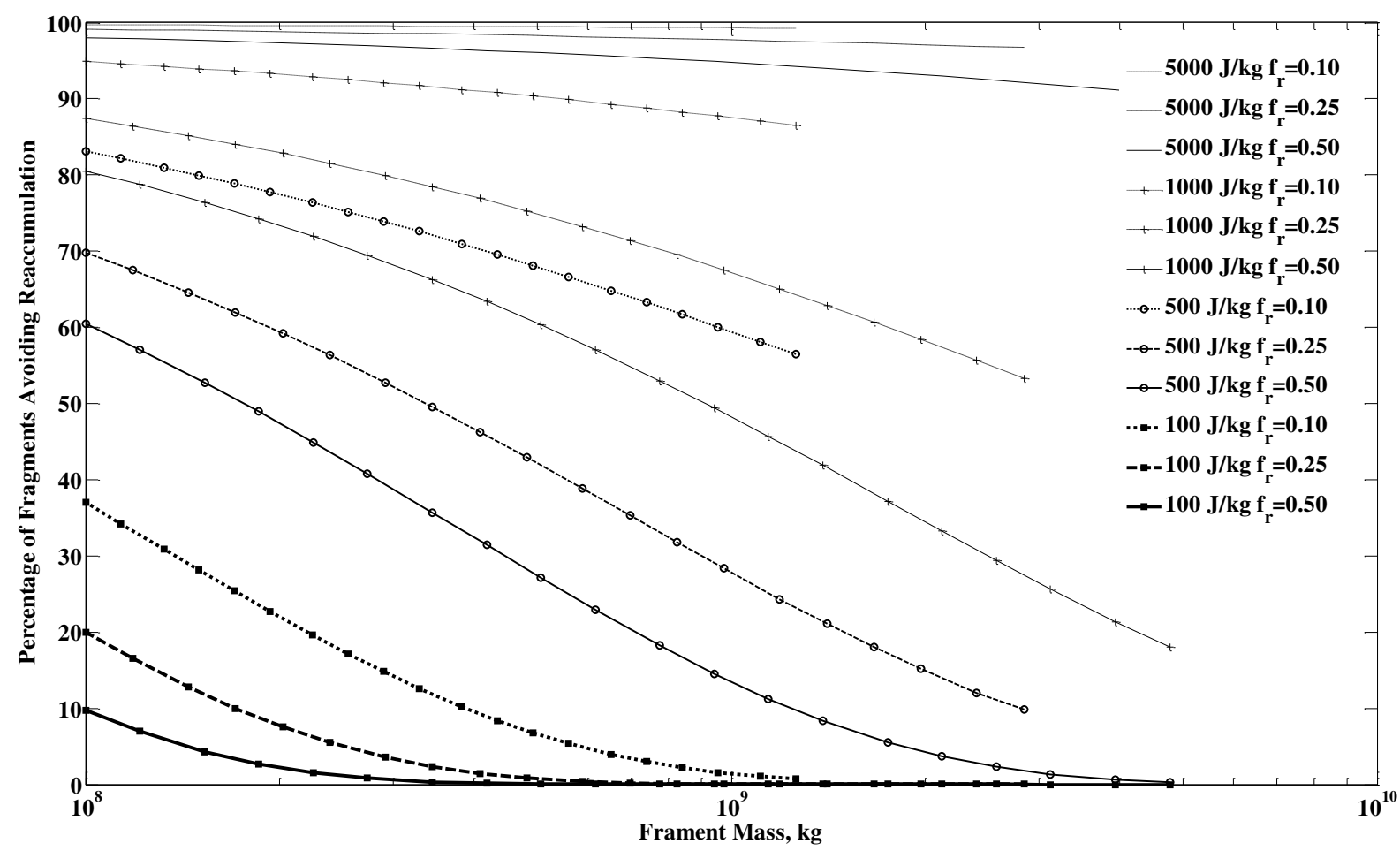

Fig. 6 Percentage of fragments escaping the gravitational field of the largest fragment and avoiding being reaggregated into the largest remaining mass of Apophis. The figure shows break-ups at four different levels of energy and three different levels of fragmentation for each level of energy.

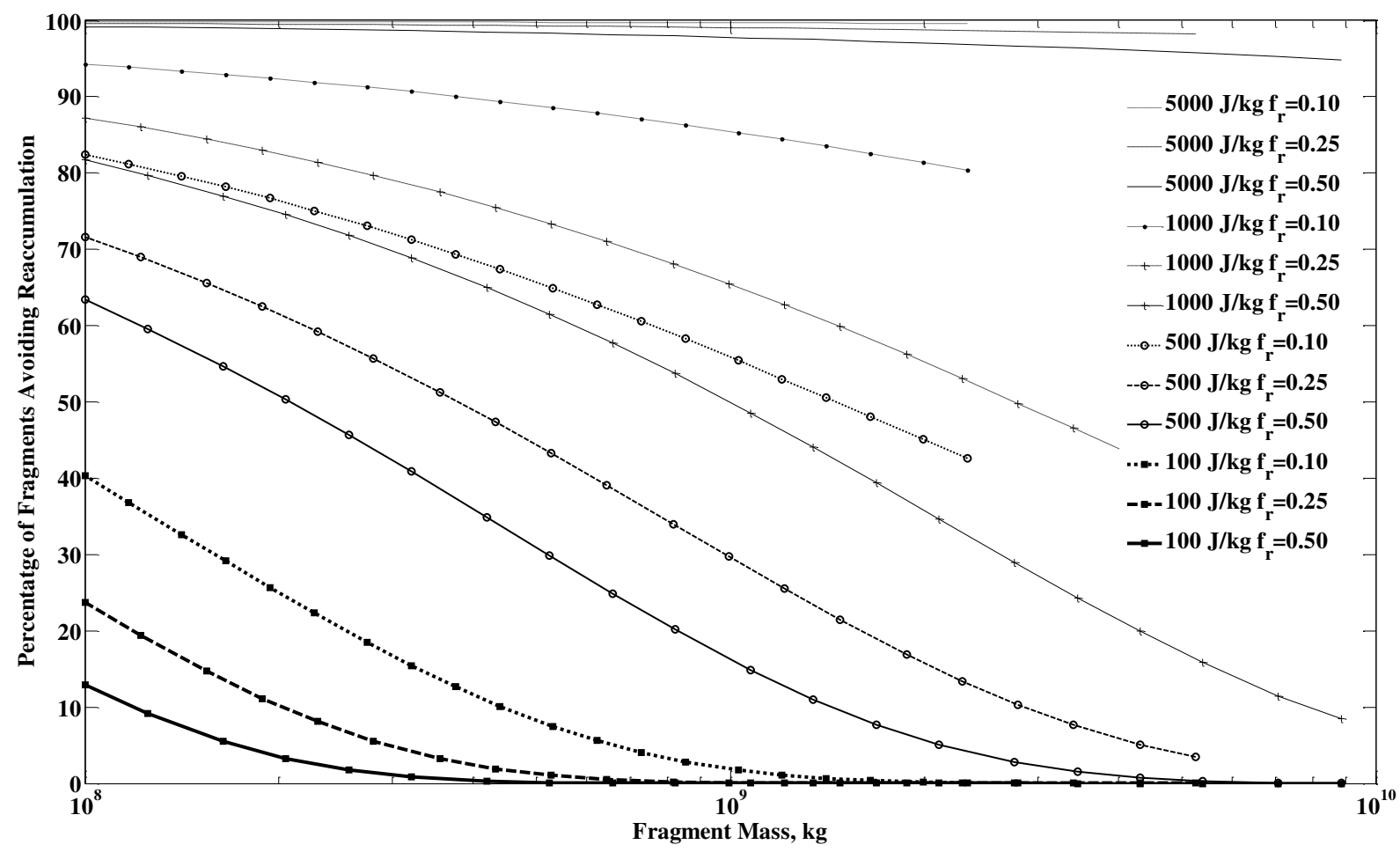

Fig. 7 Percentage of fragments escaping the gravitational field of the largest fragment for Aten and Apollo case. 


\section{Evolution of the Cloud of Fragments}

Eventually, we will assume that the number of fragments that manage to escape the largest mass' gravity well will evolve using the afore-developed statistical description of the problem. This, of course, is implicitly slowing down the velocity of the escaped fragments, since those had a relative velocity higher than the average dispersion of velocity calculated by Eq.(33). In fact, the excess mean dispersion $\boldsymbol{\sigma}$ of the escaped fragments, i.e., average velocity relative to the center of mass of the system outside the sphere of influence of the largest fragment, can be calculated and is found to differ with Eq.(33) by less than 20\%. Since the intended accuracy of the model described in Section IV is not weakened by avoiding a full gravitational analysis, this will then be neglected in this study.

In order to describe the evolution of the dispersing cloud of fragments, we can study the variation with time of the shape of the surface enclosing a certain fixed probability to find given fragment. The shape of this surface can be well described by the four features shown in Fig. 8 the semimajor axis a, the semiminor axis b, the dispersion along the $h$ axis or out-of-plane and the angle $\alpha$ between the semimajor axis a and the tangential direction axis t.

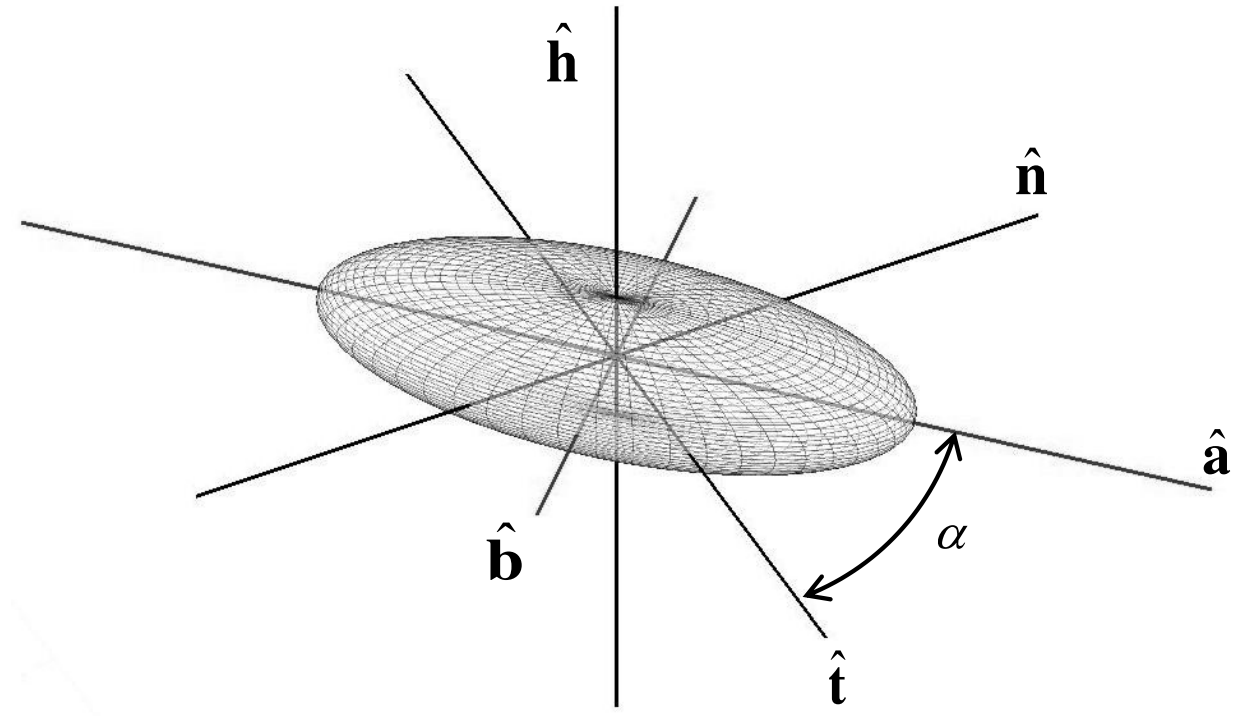

Fig. 8 Schematic of the 4 features describing the shape and attitude of the ellipsoidal shaped cloud of fragments.

Fig. 9 summarizes the evolution of the four aforementioned features along the two years following the asteroid break-up. In this example, these four features describe the volume enclosing $97 \%$ probability to find the largest fragment of a barely catastrophic fragmentation triggered after providing $500 \mathrm{~J} / \mathrm{kg}$ of collisional energy to a hypothetical $5 \times 10^{10} \mathrm{~kg}$-circular asteroid with $1 \mathrm{AU}$ of semimajor axis. A successful deflection attempt, with a collisional energy of $500 \mathrm{~J} / \mathrm{kg}$, would have provided an approximate change of velocity of $\Delta \mathrm{v}_{\mathrm{a}}=0.02 \mathrm{~m} / \mathrm{s}$, using an impactor mass $\mathrm{m}_{\mathrm{s} / \mathrm{c}}$ of $18,500 \mathrm{~kg}$. If a barely catastrophic fragmentation occurs, instead of the expected deflection and according to the model developed in Section IV, the largest fragment (i.e., $2.5 \times 10^{10} \mathrm{~kg}$ ) would have a mean velocity of $\boldsymbol{\mu}=\left[\begin{array}{lll}0.02 & 0 & 0\end{array}\right] \mathrm{m} / \mathrm{s}$ (Eq.(22)) and a standard deviation of $\boldsymbol{\sigma}=\left[\begin{array}{lll}0.012 & 0.012 & 0.012\end{array}\right] \mathrm{m} / \mathrm{s}$ (Eq.(33)). It is important to note that the evolution of the shape of the cloud is essentially driven by the dynamics of the system, which were defined by the proximal motion equations that were used to construct the transition matrix in Eq.(11). Therefore, the surface enclosing any given probability of any fragment size will follow the same pattern, only the volume enclosed would change. 


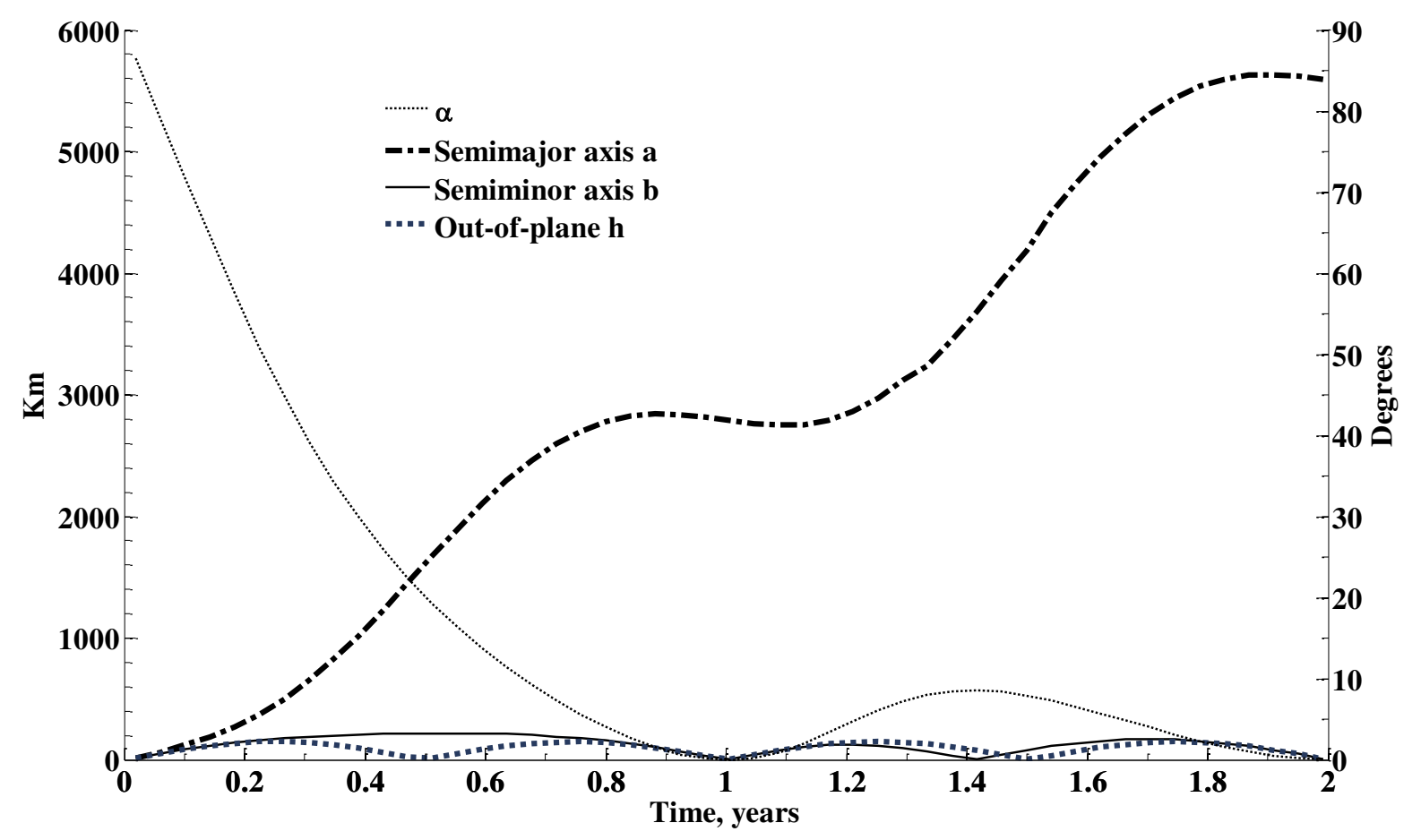

Fig. 9 Two years evolution of the four features defining the ellipsoidal cloud enclosing $97 \%$ probability to find the single $2.5 \times 10^{10} \mathrm{~kg}$ fragment.

\section{Consequences of a Fragmentation}

If the impact of each asteroid test case is assumed to occur at the MOID point for each test case listed in Table 1. the impact likelihood can be calculated by integrating over the volume inside a sphere centered at the asteroid's MOID point with radius equal to the Earth capture volume $\mathrm{dV}(\mathrm{r})$ :

$$
\mathrm{L}=\int_{\mathrm{V}(\mathrm{r}=0)}^{\mathrm{V}\left(\mathrm{r}=\varepsilon \cdot \mathrm{R}_{\oplus}\right)} \mathrm{P}\left(\mathbf{x} ;\left(\mathrm{t}_{\text {MOID }}-\mathrm{t}_{0}\right)\right) \cdot \mathrm{dV}(\mathrm{r})
$$

Note that the capture volume is approximated by the Earth radius corrected with the aforementioned hyperbolic factor $\varepsilon$, to account for the final gravitational focusing of the Earth.

From Eq.(45), we can see that the total impact likelihood for a particular fragment size and test case is only a function of the time of the closest approach $t_{\mathrm{MOID}}$, the time at which the break up occurred (the difference between these two times is here referred to as lead time) and the specific collisional energy used to break up the asteroid.Fig.| 10 shows the evolution along lead time of the impact likelihood of the largest fragment from a barely catastrophic fragmentation emanating from the hypothetical break-up of each test case presented in Table 1. In this first example, the hyperbolic factor $\varepsilon$ particular to each test is not yet taken into account and a 2.16 value, i.e., Apophis hyperbolic factor, is instead used for all the four orbits. Using the same factor $\varepsilon$ for all the test cases avoids adding third body effects (i.e., Earth final hyperbolic approach) to the problem of the dispersion of fragments, which, at this point, eases the analysis on the evolution and dispersion of the cloud of fragments. 


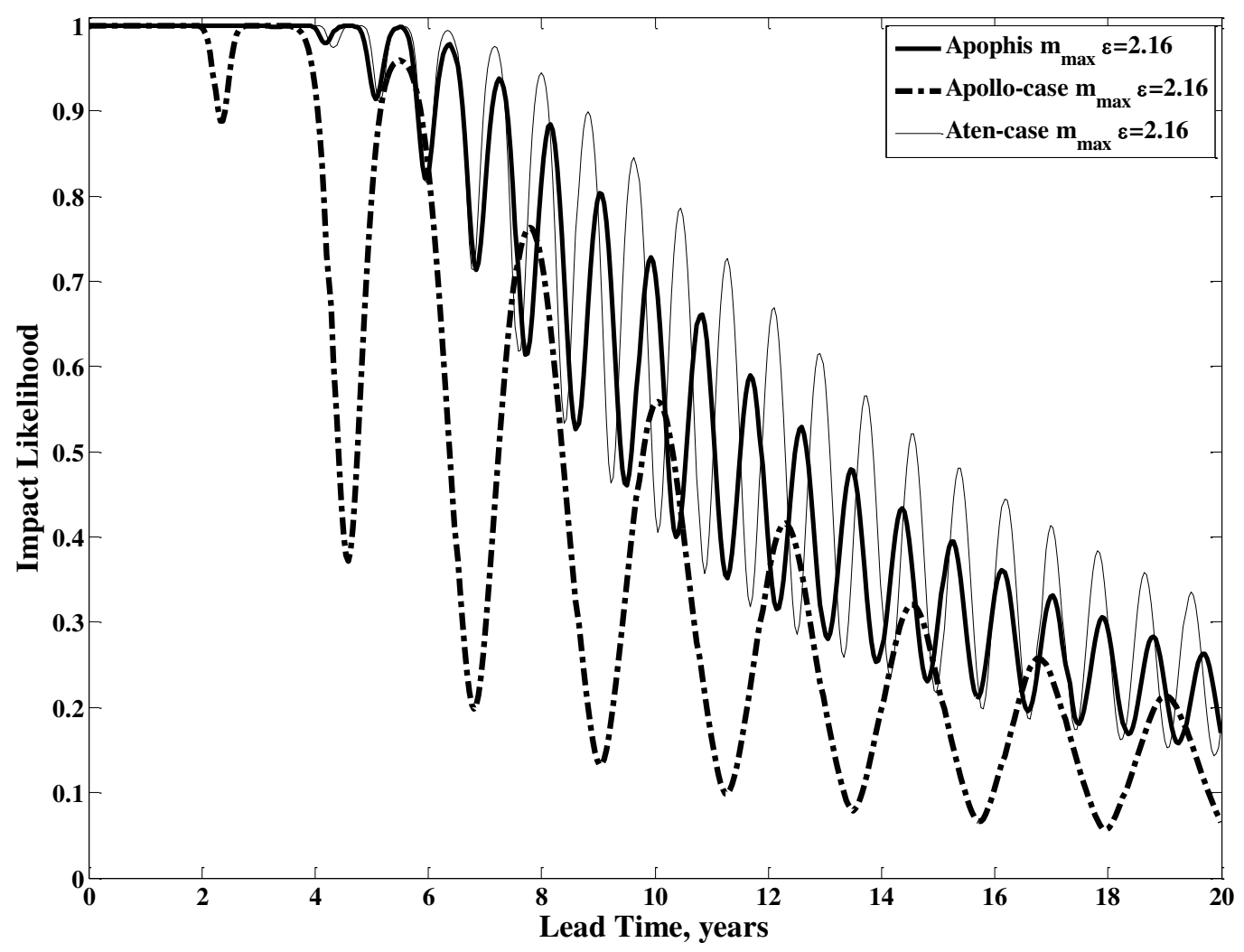

Fig. 10 Impact likelihood of the largest fragment of a barely catastrophic fragmentation for all the test cases in Table 1. The fragmentation is triggered by $500 \mathrm{~J} / \mathrm{kg}$ of collisional energy causing a dispersion of the largest fragment $\mathbf{m}_{\max }$ with $\boldsymbol{\mu}=\left[\begin{array}{lll}0.014 & 0 & 0\end{array}\right] \mathrm{m} / \mathrm{s}$ and $\boldsymbol{\sigma}=\left[\begin{array}{lll}0.008 & 0.008 & 0.008\end{array}\right] \mathrm{m} / \mathrm{s}$.

The collisional energy or SKE of the impact likelihood plotted in Fig. 10 was set at $500 \mathrm{~J} / \mathrm{kg}$. Such a collisional energy causes a change of velocity of the centre of mass of the system of $\left[\begin{array}{lll}0.014 & 0 & 0\end{array}\right] \mathrm{m} / \mathrm{s}$ and a standard deviation of the velocity of the largest fragment of $\left[\begin{array}{lll}0.008 & 0.008 & 0.008\end{array}\right] \mathrm{m} / \mathrm{s}$, which is almost $60 \%$ the $\delta \mathrm{v}$ of the centre of mass. An important difference with respect to the calculations on the evolution of the dispersion of the cloud of fragments (see Section V.C) is the fact that in Fig. 10, and in the remaining calculations in Section VI, the break up of the asteroid moves backwards in time in order to have an increase in lead time, while the hypothetical impact time $t_{M O I D}$ is kept fixed. A consequence of this is that the break up occurs at different orbital positions of the unperturbed orbit of asteroid, and the periodic variations of the impact likelihood that can be observed are primarily due to this change in orbital position of the break up point.

One of the figure's most outstanding features is the large variation of the impact likelihood within one orbital period. This variation increases with the eccentricity of each test case, hence Apollo-case is the asteroid with the largest variation of the impact likelihood within an orbit. The difference between the maximum and minimum impact probability within the same orbit reaches a maximum of $70 \%$ for the Apollo-case. Another distinctive feature of the evolution of the curves in Fig. 10 is the timing of each minimum, occurring at the perihelion position of each asteroid orbit. Both features are direct consequences of the variation of semimajor axis of the initial orbit $\delta$ a , which is the only variation of Keplerian elements that allows for an unbounded relative orbit of the fragment. The first Gauss planetary equation states:

$$
\delta \mathrm{a}=\frac{2 \mathrm{a}^{2} \mathrm{v}}{\mu} \delta \mathrm{v}_{\mathrm{t}}
$$

which, for a fixed $\delta \mathrm{v}_{\mathrm{t}}$, will be maximum at perihelion, since the orbital velocity $\mathrm{v}$ will also be highest. At the same time, the orbital variation of the velocity $\mathrm{v}$ is larger the higher the eccentricity of the asteroid, hence the orbital variation of the impact likelihood is also larger the higher the eccentricity. 


\section{A. Fragments population and Earth Impacts: $500 \mathrm{~J} / \mathrm{kg}$ case}

This section presents the impact likelihood and the average number of expected impacts for three different catastrophic fragmentations, i.e., $\mathrm{f}_{\mathrm{r}} \leq 0.5$, triggered with a $500 \mathrm{~J} / \mathrm{kg}$ kinetic impactor mission at some point during the last 20 years of collision course of Apophis, Aten and Apollo cases. In the example used here, The $500 \mathrm{~J} / \mathrm{kg}$ of collisional energy accounts for a kinetic impactor with a mass of 10,000 kg to deflect Apophis or 18,520 $\mathrm{kg}$ for the larger Aten and Apollo cases. As a consequence, the impact velocity necessary to provide the required collisional energy is $52 \mathrm{~km} / \mathrm{s}$. Such a deflection mission would provide a change of the velocity of the unfragmented asteroid (or centre of mass of the fragmented case) of $\Delta \mathbf{v}_{\mathrm{a}} \square\left[\begin{array}{lll}0.019 & 0 & 0\end{array}\right] \mathrm{m} / \mathrm{s}$.

Only a discrete number of size samples were computed due to the large computational cost of the impact likelihood integration (i.e., Eq.(45)), in particular, six different fragment masses were studied for each given case; five general representative masses at $10^{10} \mathrm{~kg}, 5 \times 10^{9} \mathrm{~kg}, 10^{9} \mathrm{~kg}, 5 \times 10^{8} \mathrm{~kg}$ and $10^{8} \mathrm{~kg}$ and a varying mass accounting for the largest fragment of each fragmentation case. As defined by Eq.(1), for the three fragmentations ratios, i.e., $f_{\mathrm{r}}$ equal to $0.5,0.25$ and 0.1 , the largest Apophis' fragment should contain a mass of $1.35 \times 10^{10} \mathrm{~kg}, 6.75 \times 10^{9} \mathrm{~kg}$ or $2.7 \times 10^{9} \mathrm{~kg}$ respectively, while for the Aten and Apollo cases the largest fragment mass should be $2.5 \times 10^{10} \mathrm{~kg}$, $1.25 \times 10^{10} \mathrm{~kg}$ or $5 \times 10^{9} \mathrm{~kg}$ for the same fragmentation ratios. Considering the re-aggregation of mass and the fragment size distribution, with a collisional energy at $500 \mathrm{~J} / \mathrm{kg}$, the largest fragments of three test cases will instead be $2.1 \times 10^{10} \mathrm{~kg}, 1.35 \times 10^{10} \mathrm{~kg}$ and $4.46 \times 10^{9} \mathrm{~kg}$ for Apophis or $4.08 \times 10^{10} \mathrm{~kg}, 2.72 \times 10^{10} \mathrm{~kg}$ and $9.68 \times 10^{9} \mathrm{~kg}$ for the other two larger cases. As we can see, the effect of the gravity is considerable, re-aggregating enough mass to yield an effective fragmentation ratio between 0.82 and 0.17 , instead of ranging from 0.50 to 0.10 . Table 2 summarizes the number of dangerous fragments considering a power law distribution such as Eq.(34) and the re-aggregation as in Section V.B.

Table 2 Fragment groups used for the computation of impact likelihood and average number of impacts for catastrophic fragmentation with $f_{r}$ equal to $0.5,0.25$ and 0.1 . Note that the smallest mass is $8 \times 10^{7} \mathrm{~kg}$, since the lower limit is set by the lower diameter limit of $40 \mathrm{~m}$. $\mathrm{N}$ is the number of average fragments rounded to the closest integer number.

\begin{tabular}{ccccccccc}
\hline & \multicolumn{3}{c}{$\mathrm{N}$} & \multicolumn{5}{c}{$\mathrm{N}$} \\
& Apophis & \multicolumn{5}{c}{ Aten- and Apollo-case } & Representative Mass \\
\hline & $\mathrm{f}_{\mathrm{r}}=$ & 0.50 & 0.25 & 0.10 & 0.50 & 0.25 & 0.10 & \\
\hline $5 \times 10^{10} \mathrm{~kg}>\mathrm{m}>1.5 \times 10^{10} \mathrm{~kg}$ & 1 & 1 & 0 & 1 & 1 & 0 & $2.5 \times 10^{10} \mathrm{~kg}$ \\
$1.5 \times 10^{10} \mathrm{~kg} \geq \mathrm{m}>7 \times 10^{9} \mathrm{~kg}$ & 0 & 0 & 1 & 0 & 0 & 1 & $1 \times 10^{10} \mathrm{~kg}$ \\
$7 \times 10^{9} \mathrm{~kg} \geq \mathrm{m}>2 \times 10^{9} \mathrm{~kg}$ & 0 & 0 & 0 & 0 & 0 & 1 & $5 \times 10^{9} \mathrm{~kg}$ \\
$2 \times 10^{9} \mathrm{~kg} \geq \mathrm{m}>7 \times 10^{8} \mathrm{~kg}$ & 1 & 1 & 1 & 1 & 2 & 2 & $1 \times 10^{9} \mathrm{~kg}$ \\
$7 \times 10^{8} \mathrm{~kg} \geq \mathrm{m}>2 \times 10^{8} \mathrm{~kg}$ & 3 & 5 & 5 & 5 & 8 & 8 & $5 \times 10^{8} \mathrm{~kg}$ \\
$2 \times 10^{8} \mathrm{~kg} \geq \mathrm{m}>8 \times 10^{7} \mathrm{~kg}$ & 8 & 11 & 11 & 12 & 19 & 18 & $1 \times 10^{8} \mathrm{~kg}$ \\
\hline \hline
\end{tabular}

As described in Section IV.B, all the fragments, no matter their mass, will have the same mean velocity, fulfilling the law of conservation of momentum, while the dispersion of velocity from the center of mass of the system is driven by Eq.(33), thus is a function of the mass of the fragment. When calculating the impact likelihood of the largest fragment, it is considered that its velocity dispersion corresponds to the dispersion of the fragment with mass equal to $f_{r} \cdot M_{a}$, so the mass of the largest fragment prior to re-aggregation process. This assumption considers that the re-aggregation process does not substantially modify the statistical description of the velocity of the largest fragment. Note that, the largest fragment will interact longer with the other fragments moving in the same direction slightly faster than the largest one (the largest is statistically the slowest); therefore, the re-aggregation is more likely to occur with those fragments. The perturbation caused by all the re-aggregating fragments will then tend to be low and in the same direction. The impact likelihood of the remaining fragments is approximated with the one associated with the closest mass range in Table 2 .

Finally, the integrated Earth capture volume of each asteroid is corrected, with the corresponding hyperbolic factor $\varepsilon$ (see Table 1), to account for the different Earth gravitational effect to each asteroid orbit. Therefore 
Apophis's likelihood integration is done inside a sphere of $2.16 \times \mathrm{R}_{\oplus}$, the Aten-case's sphere is instead $1.52 \times \mathrm{R}_{\oplus}$ and, finally, the Apollo-case's sphere is $1.29 \times \mathrm{R}_{\oplus}$.
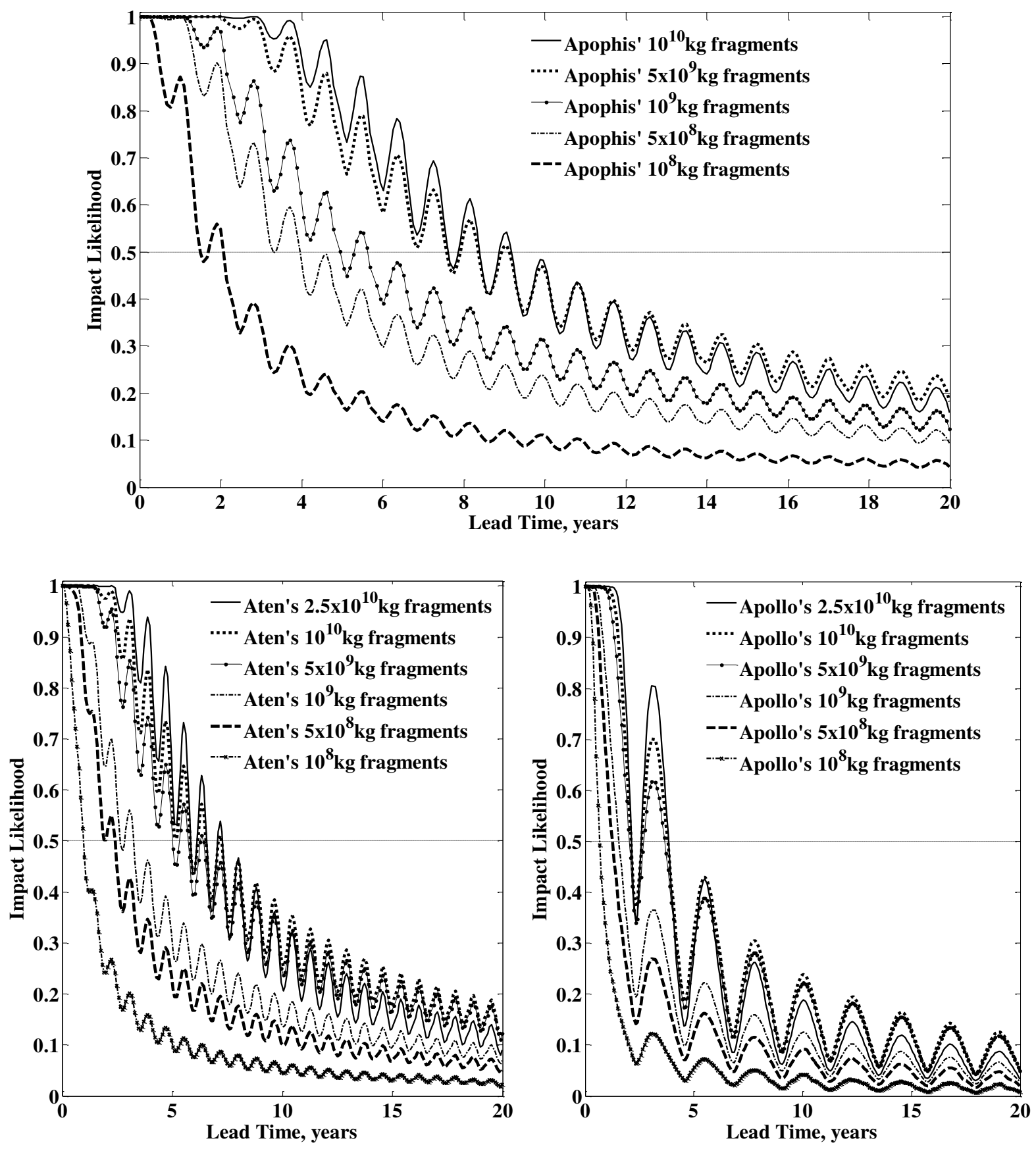

Fig. 11 Evolution of the impact likelihood for different fragment size along lead times ranging from 0 to 20 years. 

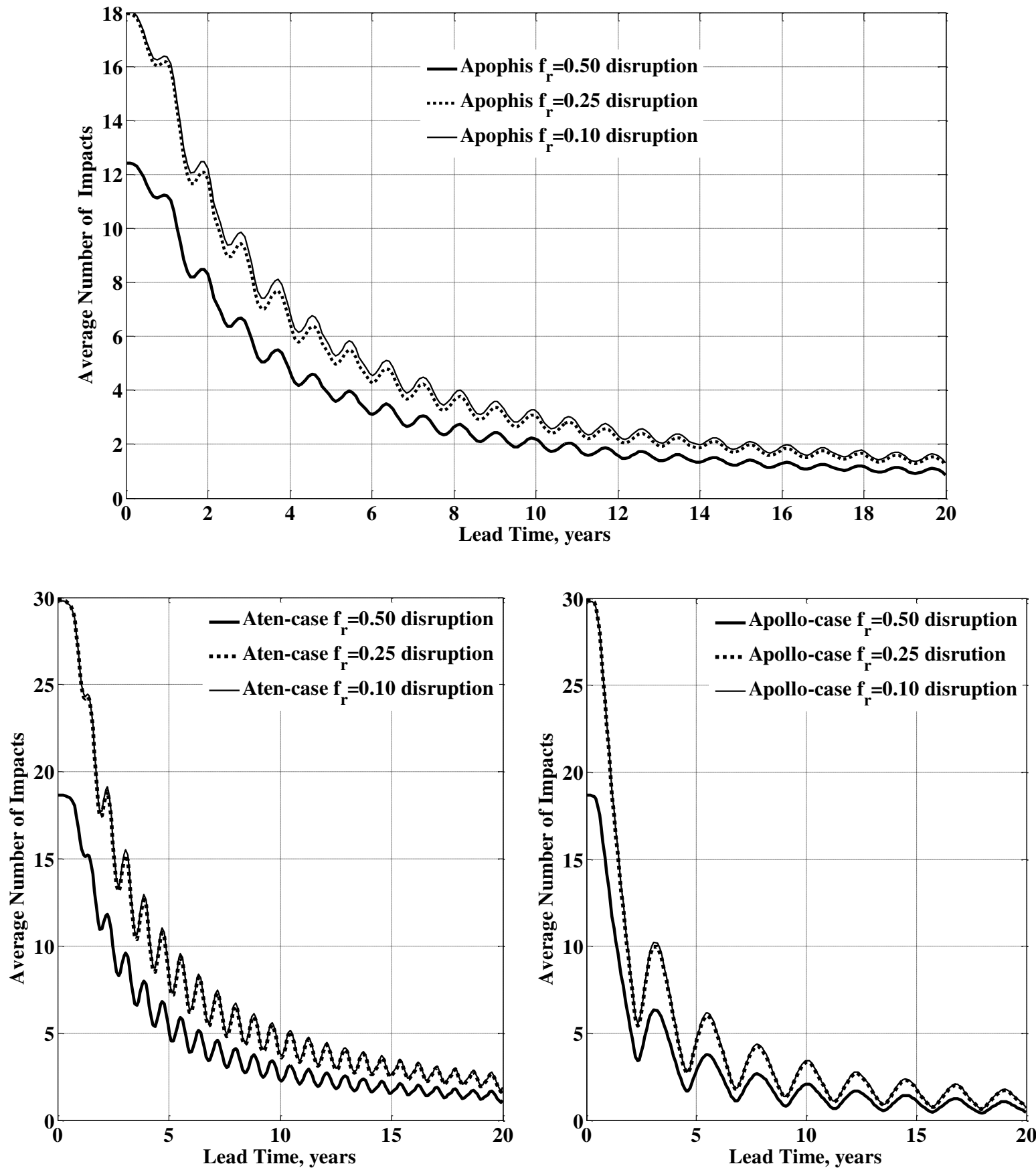

Fig. 12 Average number of impacts for three different fragmentation ratios function of the lead time.

Fig. 11 shows the evolution over lead time of the impact likelihood for the three test cases and different fragment sizes. Fig. 12 shows the average number of impacts, thus including the complete census of fragment, for the three different levels of fragmentation. As was expected, the smaller a fragment is, the quicker its impact likelihood begins to drop, which is due to the higher velocity dispersion of the smaller fragments. At a certain point, this trend changes for all fragment sizes with dispersion of velocity smaller than their mean velocity, since the centre of the ellipsoid of uncertainty moves out of the integrated volume and then the smaller the ellipsoid is the lower will be the 
impact likelihood. Despite that, in general, the impact likelihood decreases with a decreasing mass, the number of expected impacts grows with a decaying mass and, as seen in Fig. 12, even if the break-up occurred 20 years in advance a few impacts should still be expected.

\section{B. Expected Damage: $500 \mathrm{~J} / \mathrm{kg}$ case}

As shown in Fig. 12, if the outcome of a deflection attempt is the catastrophic break-up of the threatening object, several impacts of small fragments could be expected even if the fragmentation or break-up occurred 20 years prior to the forecasted impact. Nevertheless, the number of expected impacts is not a good figure to evaluate the risk that these small objects pose to Earth. The work of Hills and Goda ${ }^{[16]}$ and Chesley and Ward ${ }^{[18]}$ will be used to assess the damage that these smaller fragments can cause and, finally, the damage will be compared with the initial damage that the unshattered object would have caused.

Clearly, an asteroid or fragment threatening to impact the Earth would have $2 / 3$ chances to fall into the water and only $1 / 3$ to fall into land. A small land impact tends to be much more localized than a sea impact, since water can transmit the impact energy over very large distances on two-dimensional waves. Adding to the efficient energy propagation, the high coastal density population makes water impacts a major element of the impact hazard.

The next three tables Table 3 to Table 5) shows the expected damage for both the unshattered objects and each one of the fragment sizes analysed earlier. Land damage is assessed using Hills and Goda ${ }^{[16]}$ 's calculations; for all fragments size, the radius of destruction is taken from the worse case between soft and hard stone of a $20 \mathrm{~km} / \mathrm{s}$ impact. Water damage, instead, is evaluated using data accounting also for $20 \mathrm{~km} / \mathrm{s}$ water impacts found in Stokes et $\mathrm{al}^{[20]}$., which were computed using the assessment on damage generated by tsunamis from Chesley and Ward ${ }^{[18]}$. Since the impact velocity of the three test cases analysed here differs from $20 \mathrm{~km} / \mathrm{s}$ (see Table 1), the predicted areas were scaled by the collisional energy fraction to the power of $2 / 3$, which is believed to be the way that explosive devastation area scales with the energy ${ }^{[35]}$.

Table 3 Expected damaged area for Apophis. Table summarizes the damage for the unshattered object and its fragments, including the aggregated largest fragments for break-ups with $f_{\mathrm{r}}$ at $0.50,0.25$ and 0.10 .

\begin{tabular}{ccccc}
\hline \hline Mass & Diameter & $\begin{array}{c}\text { Land Damage } \\
\text { Area } \\
{\left[\mathrm{km}^{2}\right]}\end{array}$ & $\begin{array}{c}\text { Water Damage } \\
\text { Area }\end{array}$ & $\begin{array}{c}\text { Weighted Damage } \\
\text { Area }\end{array}$ \\
& & $\sim 5,920$ & $\sim 56,940$ & {$\left[\mathrm{~km}^{2}\right]$} \\
\hline $2.7 \times 10^{10} \mathrm{~kg}$ & $270 \mathrm{~m}$ & $\sim 5,253$ & $\sim 44,340$ & $\sim 39,930$ \\
$2.1 \times 10^{10} \mathrm{~kg}^{\mathrm{fr}=0.50}$ & $250 \mathrm{~m}$ & $\sim 4,429$ & $\sim 30,000$ & $\sim 21,477$ \\
$1.35 \times 10^{10} \mathrm{~kg}{ }^{\mathrm{fr}=0.25}$ & $215 \mathrm{~m}$ & $\sim 2,988$ & $\sim 7,719$ & $\sim 6,142$ \\
$4.46 \times 10^{9} \mathrm{~kg} \mathrm{fr}=0.10$ & $149 \mathrm{~m}$ & $\sim 2,080$ & $\sim 240$ & $\sim 860$ \\
$1 \times 10^{9} \mathrm{~kg}$ & $90 \mathrm{~m}$ & $\sim 750$ & $\sim 40$ & $\sim 280$ \\
$5 \times 10^{8} \mathrm{~kg}$ & $71 \mathrm{~m}$ & $\sim 40$ & $\sim 0$ & $\sim 10$ \\
$1 \times 10^{8} \mathrm{~kg}$ & $42 \mathrm{~m}$ & & & \\
\hline \hline
\end{tabular}


Table 4 Aten-case's expected damaged area.

\begin{tabular}{|c|c|c|c|c|}
\hline Mass & Diameter & $\begin{array}{c}\text { Land Damage } \\
\text { Area } \\
{\left[\mathrm{km}^{2}\right]}\end{array}$ & $\begin{array}{c}\text { Water Damage } \\
\text { Area } \\
{\left[\mathrm{km}^{2}\right]}\end{array}$ & $\begin{array}{c}\text { Weighted Damage } \\
\text { Area } \\
{\left[\mathrm{km}^{2}\right]}\end{array}$ \\
\hline $5 \times 10^{10} \mathrm{~kg}$ & $332 \mathrm{~m}$ & $\sim 11,260$ & $\sim 110,770$ & $\sim 77,600$ \\
\hline $4.1 \times 10^{10} \mathrm{~kg}^{\mathrm{fr}=0.50}$ & $311 \mathrm{~m}$ & $\sim 9,959$ & $\sim 93,650$ & $\sim 65,632$ \\
\hline $2.7 \times 10^{10} \mathrm{~kg}^{\mathrm{fr}=0.25}$ & $271 \mathrm{~m}$ & $\sim 7,390$ & $\sim 71,110$ & $\sim 49,867$ \\
\hline $9,68 \times 10^{9} \mathrm{~kg}^{\mathrm{fr}=0.10}$ & $192 \mathrm{~m}$ & $\sim 4,990$ & $\sim 29,020$ & $\sim 21,011$ \\
\hline $5 \times 10^{9} \mathrm{~kg}$ & $154 \mathrm{~m}$ & $\sim 3,910$ & $\sim 12,200$ & $\sim 9,440$ \\
\hline $1 \times 10^{9} \mathrm{~kg}$ & $90 \mathrm{~m}$ & $\sim 2,590$ & $\sim 300$ & $\sim 1,070$ \\
\hline $5 \times 10^{8} \mathrm{~kg}$ & $71 \mathrm{~m}$ & $\sim 930$ & $\sim 50$ & $\sim 350$ \\
\hline $1 \times 10^{8} \mathrm{~kg}$ & $42 \mathrm{~m}$ & $\sim 50$ & $\sim 0$ & $\sim 20$ \\
\hline
\end{tabular}

Table 5 Apollo-case's expected damaged area.

\begin{tabular}{ccccc}
\hline \hline Mass & Diameter & $\begin{array}{c}\text { Land Damage } \\
\text { Area } \\
{\left[\mathrm{km}^{2}\right]}\end{array}$ & $\begin{array}{c}\text { Water Damage } \\
\text { Area } \\
{\left[\mathrm{km}^{2}\right]}\end{array}$ & $\begin{array}{c}\text { Weighted Damage } \\
\text { Area } \\
{\left[\mathrm{km}^{2}\right]}\end{array}$ \\
\hline $5 \times 10^{10} \mathrm{~kg}$ & $332 \mathrm{~m}$ & $\sim 14,310$ & $\sim 140,830$ & $\sim 98,660$ \\
$4.1 \times 10^{10} \mathrm{~kg}^{\mathrm{r}=0.50}$ & $311 \mathrm{~m}$ & $\sim 12,200$ & $\sim 119,070$ & $\sim 83,443$ \\
$2.7 \times 10^{10} \mathrm{~kg}^{\mathrm{r}=0.25}$ & $271 \mathrm{~m}$ & $\sim 9,390$ & $\sim 90,400$ & $\sim 63,400$ \\
$9,68 \times 10^{9} \mathrm{~kg}^{\mathrm{fr}=0.10}$ & $192 \mathrm{~m}$ & $\sim 6,350$ & $\sim 36,890$ & $\sim 26,713$ \\
$5 \times 10^{9} \mathrm{~kg}$ & $154 \mathrm{~m}$ & $\sim 4,970$ & $\sim 15,510$ & $\sim 12,000$ \\
$1 \times 10^{9} \mathrm{~kg}$ & $90 \mathrm{~m}$ & $\sim 3,290$ & $\sim 390$ & $\sim 1,350$ \\
$5 \times 10^{8} \mathrm{~kg}$ & $71 \mathrm{~m}$ & $\sim 1,180$ & $\sim 70$ & $\sim 440$ \\
$1 \times 10^{8} \mathrm{~kg}$ & $42 \mathrm{~m}$ & $\sim 70$ & $\sim 0$ & $\sim 20$ \\
\hline \hline
\end{tabular}

Hills and Goda ${ }^{[16]}$ estimated that asteroidal bolides larger than a few tens of meters in diameter are already able to cause damage to the Earth surface, although only due to the sudden blast produced in the final moments of the dissipation of the bolide when crossing the Earth atmosphere. This will not leave long lasting scars on the surface, but only cause an atmospheric explosion like the one occurred in Tunguska (Siberia) in $1908^{[15]}$. Instead, bolides above $150-200 \mathrm{~m}$ in diameter ${ }^{[16 ; 17]}$ reach the Earth surface producing cratering events and, if falling into the sea, dangerous tsunamis ${ }^{[18]}$. Note from the tables above, the sudden reduction in water damaged area below the $150 \mathrm{~m}$ diameter. This is due to the low efficiency of air blasts in transmitting their energy to the ocean surface in order to initiate a tsunami.

We also considered a weighted damage ratio. The weighted damage ratio considers the mean damage of a statistical distribution of land and water impacts. One could think that although for small fragments the number of impacts is high enough to make the weighted damage a good approximation, for the largest fragments and especially for the unfragmented asteroid the approximation can drive to misleading results, since a single fragment would not cause a weighted damage, but one of the two options, i.e., either land or water impact.

Let us suppose that a fragmentation spawning several big fragments (i.e., $>5 \times 10^{9} \mathrm{~kg}$ ) occurs. This kind of fragmentation outcome would be of very rare occurrence when triggered by only $500 \mathrm{~J} / \mathrm{kg}$ of SKE, but may happen more often for higher collisional energies. If several large fragments are then spawned by the break-up of an asteroid, the most worrying scenario would occur if the unshattered object was meant to have a land impact, but 
because of the failed attempt to mitigate the threat, at least 1 of the large fragments fall into the sea. This scenario would yield more damage to Earth than previous unfragmented scenario and, considering each fragment as statistically independent, would occur with little less than 33\% probability (if having several large objects).On the other hand, if the unshattered object is meant to hit the sea, only the very infrequently occurring scenario of several large objects, which manage not to become re-aggregated, and all of them falling into the water could possibly increase the damage caused by the tsunami produced by the single unshattered object. To sum up, there is only a little more than $33 \%$ probability to increase the damage by fragmentation of the original asteroid, if both the unshattered object and all its fragments fall onto Earth. Highlighting the latter result, the statistical weighted damage is used on the rest of the analysis of consequences of a fragmentation.

Fig. 13 shows the evolution with lead time of the damage probability of different sizes produced by a barely catastrophic fragmentation, i.e., $\mathrm{f}_{\mathrm{r}}=0.50$, of Apophis. We refer here as damage probability the potential damage of a given fragment size multiplied by the probability of impact of the fragments of the same size. As shown in the figure, despite an increasing number of fragments, the statistical damaged area drops with a decreasing fragment mass, being the largest fragment clearly the most dangerous of all, although even the smallest fragments, $10^{8} \mathrm{~kg}$, may still cause considerable damage.

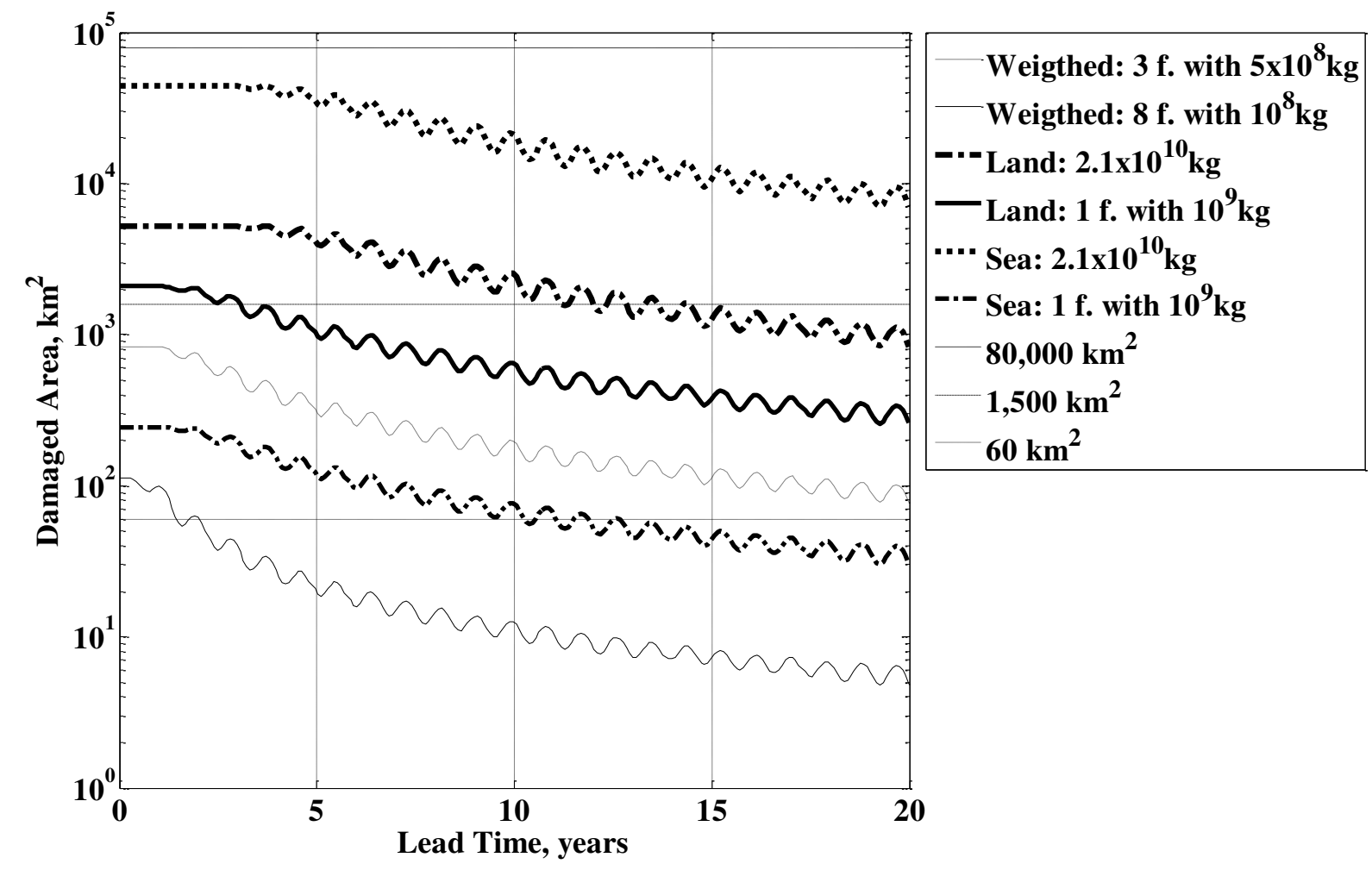

Fig. 13 Damage evolution of a barely catastrophic fragmentation of Apophis. Sizes with more than one fragment use the weighted damage, while sizes with only one representative use both land and sea damage. For comparison, the three straight lines represent the area of Scotland $\left(\sim 80,000 \mathrm{~km}^{2}\right)$, the area of London $\left(\sim 1,500 \mathrm{~km}^{2}\right)$ and the area of Manhattan $\left(\sim 60 \mathrm{~km}^{2}\right)$.

Fig. 14 compares the damage probability of the unshattered Apophis with the total damage probability of the three different catastrophic fragmentation levels, i.e., $\mathrm{f}_{\mathrm{r}}$ at $0.5,0.25$ and 0.1 . The damage of the three fragmented cases is computed by adding the predicted Apophis's weighted damage of each fragment size, thus multiplying the weighted damaged areas in Table 3 by the number of expected impacts of each fragment size previously calculated and shown in Fig. 12. The computed damage probability in all the following figures has been scaled by the weighted damage of the unfragmented Apophis, $\sim 40,000 \mathrm{~km}^{2}$, and will be referred as damage ratio. The fragmentation plotted in Fig. 14 was triggered by a kinetic impactor with $\mathrm{m}_{\mathrm{s} / \mathrm{c}}$ of $10,000 \mathrm{~kg}$ providing $500 \mathrm{~J} / \mathrm{kg}$ of SKE. If Apophis does not 
shatter under such a collisional energy the asteroid could be deflected with a velocity of $\boldsymbol{\mu} \square\left[\begin{array}{lll}0.019 \mathrm{~m} / \mathrm{s} \quad & 0 & 0\end{array}\right]$, assuming an enhancement factor $\beta$ of only 1 .

As seen in Fig. 14, the unshattered Apophis completely misses the Earth 12 years after its orbit was altered by $0.019 \mathrm{~m} / \mathrm{s}$, while within 6 to 12 years of lead time, Apophis misses the Earth only if the deflection occurs at several optimal orbital positions. The damage ratio of the unshattered object (thin solid line) was computed not only by applying a delta-velocity $\delta \mathbf{v} \square[0.019 \mathrm{~m} / \mathrm{s} \quad 0 \quad 0]$ to Apophis, but also adding a small error to account for sensible uncertainties during the mitigation mission. A standard deviation $\sigma_{0}$ of $1 / 6$ th the delta-velocity is chosen as a generic error for all the unfragmented computations, this standard deviation states that, after taking into account the uncertainties of the model, the ultimate value of the delta-velocity has a $99.7 \%$ of probability to be within $50 \%$ the value predicted by the model, thus:

$$
3 \sigma_{0}=\frac{\Delta \mathrm{v}_{\mathrm{a}}}{2}
$$

Without this hypothetical error in the kinetic impactor performance, the damage ratio (thin solid line) would simply resemble a step function.

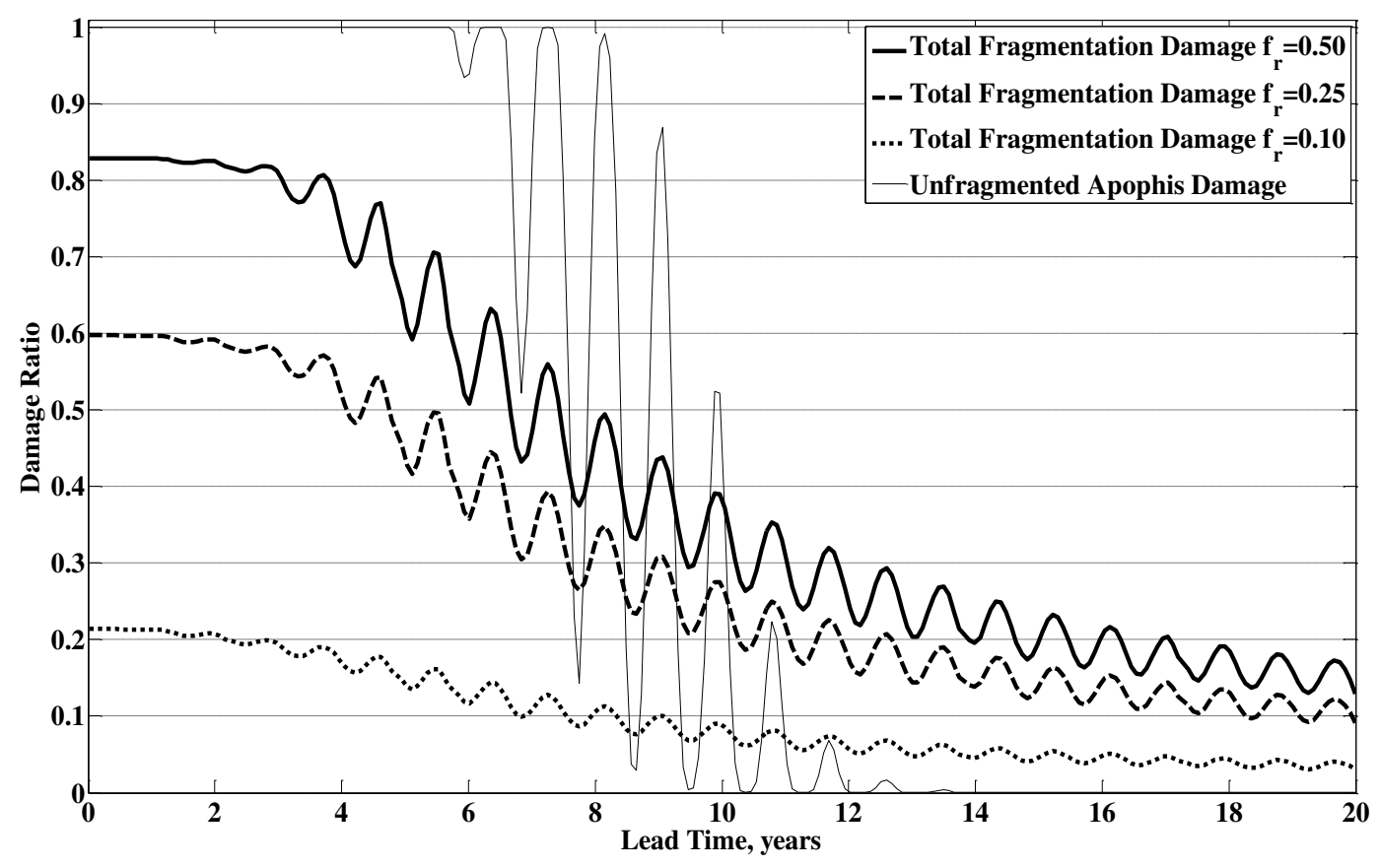

Fig. 14 Total fragmentation damage ratio of Apophis: fragmented case $f_{r}=0.50$ (black solid line), $f_{r}=0.25$ (black dashed line), $\mathrm{f}_{\mathrm{r}}=\mathbf{0 . 1 0}$ (black dotted line) and unshattered case (thin black line) with a tree sigma equal to $50 \%$ in the delta-velocity.

Fig. 15 completes the comparison on the consequences of a fragmentation for the Aten and Apollo-cases with SKE of $500 \mathrm{~J} / \mathrm{kg}$. The unshattered damage for these two objects is also calculated using the same generic error described above. Fig. 14 and Fig. 15 highlight several interesting features on the consequences of a catastrophic fragmentation; if a fragmentation occurs, the maximum damage, i.e., the damage that would be caused by all fragments impacting the Earth, is smaller than that of the unshattered object, and keeps decreasing with a decreasing fragmentation ratio. There is however an exception to this: as we can see by using the data available from Table 2 to Table 5, several land impacts will easily cause more harm that the unshattered land damage, while the opposite occurs for sea impacts. 

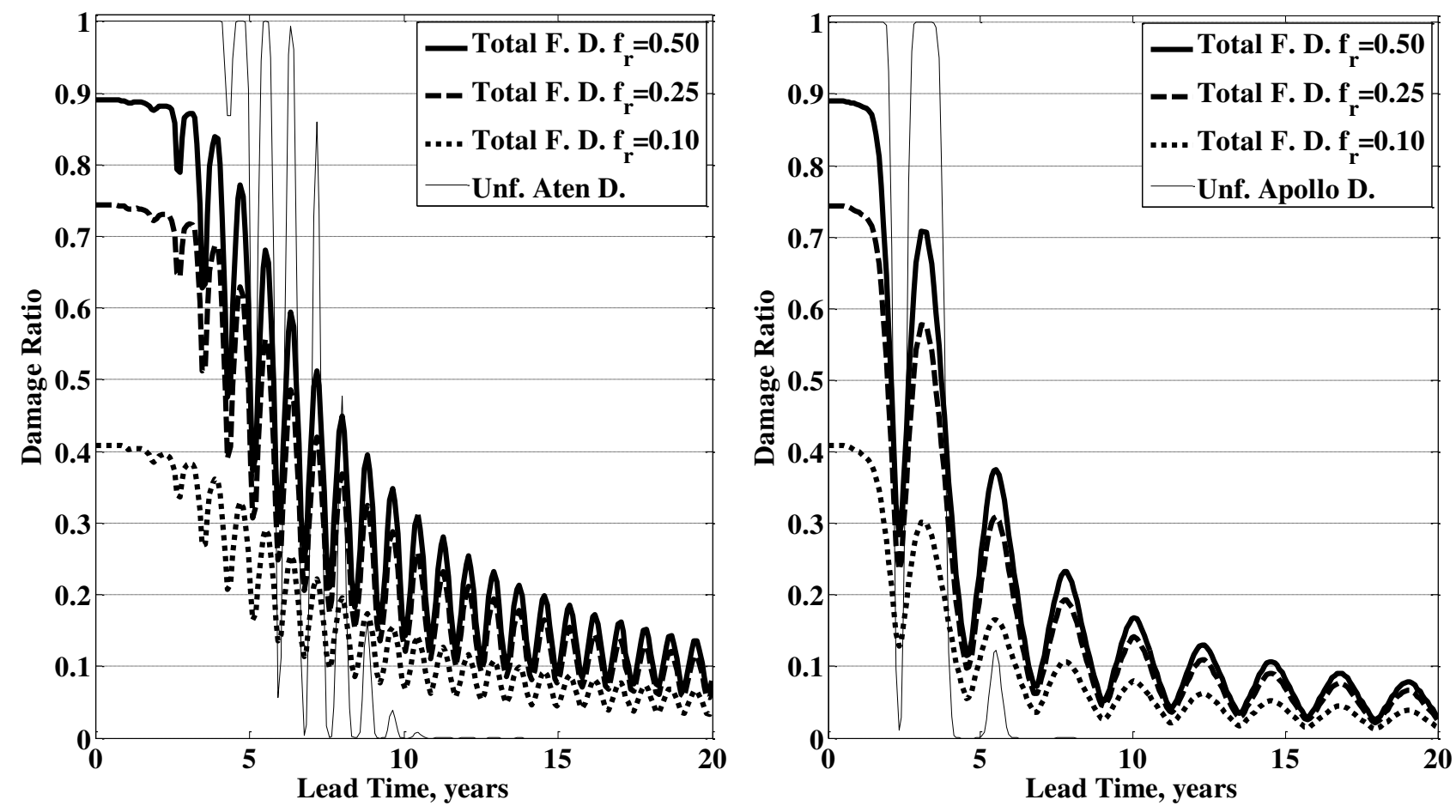

Fig. 15 Total fragmentation damage ratios (F.D.) of the Aten and Apollo-cases.

One of the most outstanding features from Fig. 14 and Fig. 15 is the higher damage ratio of all the fragmented scenarios opposed to the zero damage of the unshattered case for long lead times ( $>10$ years). We should notice from Fig. 13 that, at this level of SKE, the damage is driven by the largest fragment: on the one hand, since the potential damage of the largest fragment is smaller than the unshattered object, the risk should be reduced, although on the other hand, the orbital uncertainty associated with the fragmentation greatly enhances the risk. Notice from Fig. 2 that the delta-velocities required to deflect the collisional course of a threatening object vary very little for long warning times, being the uncertainties associated with the fragmentation much larger than this variation.

Finally, in order to provide some insight into the robustness of the model, Fig.16 compares the nominal barely catastrophic fragmentation of Apophis against four different variations of some of the most important parameters of the model; the density of the asteroid, the exponent x from Eq.(29) and the constant k from Eq.(31). The largest differences among the five test cases in the figure are found at very short lead times, where impact probability is 1 , or close, and the differences in damage ratio are driven by the differences in re-accumulated mass for each simulation. For long warning times, instead, the differences are small and are driven, in all cases, by the slow dumping of the remaining impact probability. This behavior is also seen in Fig. 14 and Fig. 15, where the difference between the three fragmentation ratios is only remarkable for short lead times. 


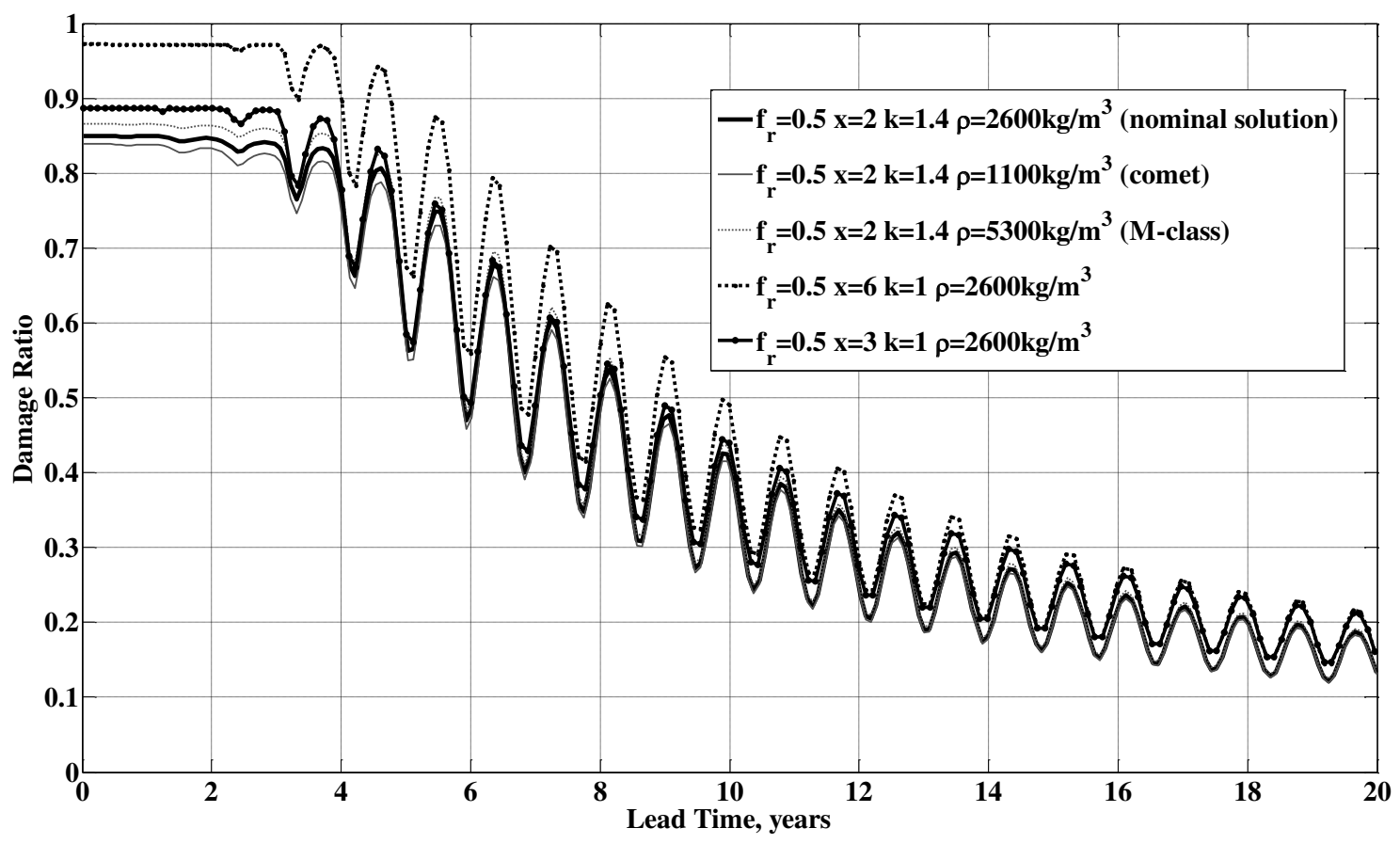

Fig. 16 Comparison of the evolution of the Damage Ratio of the nominal solution. Two extreme densities (comet and M-class asteroid) and two higher exponent $x$ as suggested by the results of Nakamura et al. ${ }^{[29]}$ were simulated and compared with the nominal model set up for a barely catastrophic fragmentation (i.e., $\left.f_{r}=0.5\right)$ of Apophis.

\section{Other Analyzed Scenarios}

The following points from the break-up and dispersion model described previously should be highlighted:

- For a fixed impactor mass $\mathrm{m}_{\mathrm{s} / \mathrm{c}}$, the delta-velocity provided to the centre of mass of the asteroid is a function only of the collisional energy or SKE used during the mitigation attempt.

- The dispersion of the cloud of fragments of a given size is a function only of the delta-velocity provided to the centre of mass.

- The potential damage that a fragmented asteroid could cause to Earth is therefore a function only of two variables; the delta-velocity provided to the centre of mass of the asteroid, which depends only on the SKE, and the fragmentation ratio of the break-up.

- A barely catastrophic fragmentation, i.e., $\mathrm{f}_{\mathrm{r}}$ equal to 0.5 , only occurs if the $\mathrm{SKE}$ (or $\mathrm{SNE}$ ) is equal to $\mathrm{Q}^{*}$, otherwise if the SKE(or SNE) is larger than $Q^{*}$ the fragmentation ratio $f_{r}$ will be smaller.

- The critical specific energy $\mathrm{Q}^{*}$ is uncertain; possibly close to $100 \mathrm{~J} / \mathrm{kg}$ for "rubble piles" and still below $1000 \mathrm{~J} / \mathrm{kg}$ even for strong monolithic asteroids.

Up to this point in the paper, we have only analyzed the consequences of a $500 \mathrm{~J} / \mathrm{kg}$ impact triggering three different levels of fragmentation and, given the aforementioned statements about the model, a few more possible scenarios should also be assessed in order to provide a good insight to the developed models and to the possible consequences of a fragmentation. The following scenarios are analyzed in this section; a barely catastrophic fragmentation triggered by a very low level of collisional energy $(100 \mathrm{~J} / \mathrm{kg})$, different level of catastrophic fragmentation triggered by the upper limit of collisional energy $(1000 \mathrm{~J} / \mathrm{kg}$ ) and, finally, a highly catastrophic fragmentation achieved with an energy much higher than the upper fragmentation limit to account for the possibility of a hazard mitigation mission attempting to destroy and disperse the impact threat as oppose to deviate it. Table 6 summarises all the scenarios used throughout the paper. 
Table 6 Summary of all fragmentation scenarios simulated throughout the paper.

\begin{tabular}{|c|c|c|c|c|}
\hline & $100 \mathrm{~J} / \mathrm{kg}$ & $500 \mathrm{~J} / \mathrm{kg}$ & $1000 \mathrm{~J} / \mathrm{kg}$ & $5000 \mathrm{~J} / \mathrm{kg}$ \\
\hline \multirow[t]{6}{*}{ Apophis } & Kinetic Impactor & Kinetic Impactor & Kinetic Impactor & Nuclear Interceptor \\
\hline & $\Delta \mathbf{v}_{\mathrm{a}} \square$ & $\Delta \mathbf{v}_{\mathrm{a}} \square$ & $\Delta \mathbf{v}_{\mathrm{a}} \square$ & $\Delta \mathbf{v}_{\mathrm{a}} \square$ \\
\hline & {$\left[\begin{array}{lll}0.006 & 0 & 0\end{array}\right] \mathrm{m} / \mathrm{s}$} & {$\left[\begin{array}{lll}0.019 & 0 & 0\end{array}\right] \mathrm{m} / \mathrm{s}$} & {$\left[\begin{array}{lll}0.038 & 0 & 0\end{array}\right] \mathrm{m} / \mathrm{s}$} & {$\left[\begin{array}{lll}0.161 & 0 & 0\end{array}\right] \mathrm{m} / \mathrm{s}$} \\
\hline & $\mathrm{m}_{\mathrm{s} / \mathrm{c}}=5,000 \mathrm{~kg}$ & $\mathrm{~m}_{\mathrm{s} / \mathrm{c}}=10,000 \mathrm{~kg}$ & $\mathrm{~m}_{\mathrm{s} / \mathrm{c}}=20,000 \mathrm{~kg}$ & $\mathrm{~m}_{\mathrm{s} / \mathrm{c}}=550 \mathrm{~kg}$ \\
\hline & $\Delta \mathbf{v}_{\mathrm{s} / \mathrm{c}}$ & $\Delta \mathbf{v}_{\mathrm{s} / \mathrm{c}} \square$ & $\Delta \mathbf{v}_{\mathrm{s} / \mathrm{c}} \square$ & \\
\hline & {$\left[\begin{array}{lll}33 & 0 & 0\end{array}\right] \mathrm{km} / \mathrm{s}$} & {$\left[\begin{array}{lll}52 & 0 & 0\end{array}\right] \mathrm{km} / \mathrm{s}$} & {$\left[\begin{array}{lll}52 & 0 & 0\end{array}\right] \mathrm{km} / \mathrm{s}$} & \\
\hline \multirow{6}{*}{$\begin{array}{l}\text { Aten and } \\
\text { Apollo } \\
\text { cases }\end{array}$} & Kinetic Impactor & Kinetic Impactor & Kinetic Impactor & Nuclear Interceptor \\
\hline & $\Delta \mathbf{v}_{\mathrm{a}} \square$ & $\Delta \mathbf{v}_{\mathrm{a}} \square$ & $\Delta \mathbf{v}_{\mathrm{a}} \square$ & $\Delta \mathbf{v}_{\mathrm{a}} \square$ \\
\hline & {$\left[\begin{array}{lll}0.006 & 0 & 0\end{array}\right] \mathrm{m} / \mathrm{s}$} & {$\left[\begin{array}{lll}0.019 & 0 & 0\end{array}\right] \mathrm{m} / \mathrm{s}$} & {$\left[\begin{array}{lll}0.038 & 0 & 0\end{array}\right] \mathrm{m} / \mathrm{s}$} & {$\left[\begin{array}{lll}0.165 & 0 & 0\end{array}\right] \mathrm{m} / \mathrm{s}$} \\
\hline & $\mathrm{m}_{\mathrm{s} / \mathrm{c}}=9,260 \mathrm{~kg}$ & $\mathrm{~m}_{\mathrm{s} / \mathrm{c}}=18,520 \mathrm{~kg}$ & $\mathrm{~m}_{\mathrm{s} / \mathrm{c}}=37,040 \mathrm{~kg}$ & $\mathrm{~m}_{\mathrm{s} / \mathrm{c}}=1,020 \mathrm{~kg}$ \\
\hline & $\Delta \mathbf{v}_{\mathrm{s} / \mathrm{c}} \square$ & $\Delta \mathbf{v}_{\mathrm{s} / \mathrm{c}} \square$ & $\Delta \mathbf{v}_{\mathrm{s} / \mathrm{c}} \square$ & \\
\hline & {$\left[\begin{array}{lll}33 & 0 & 0\end{array}\right] \mathrm{km} / \mathrm{s}$} & {$\left[\begin{array}{lll}52 & 0 & 0\end{array}\right] \mathrm{km} / \mathrm{s}$} & {$\left[\begin{array}{lll}52 & 0 & 0\end{array}\right] \mathrm{km} / \mathrm{s}$} & \\
\hline
\end{tabular}

Barely catastrophic fragmentation with $100 \mathrm{~J} / \mathrm{kg}$

An SKE of $100 \mathrm{~J} / \mathrm{kg}$ provides very little velocity to the threatening object as can be seen in Fig. 17, where the impulse provided to the three unshattered object is barely able to deflect the Apollo-case. On the other hand, if the asteroid shatters, the fragments will have very little velocity and most of the mass will re-accumulate. As described in Table 7, less than $10 \%$ of the mass is predicted to escape the re-accumulation process, thus the potential risk of damage comes almost entirely from the large re-accumulated fragment. There is a small initial reduction on the damage caused by the fragmented cases, caused by the mass loss, since all the small fragments that do manage to escape the gravitational re-accumulation are, in fact, too small to reach the Earth surface and cause any noticeable damage. We can notice however a higher damage on the fragmented case for long lead times in the Apollo-case's figure, which is caused by the increased uncertainty on the motion of the new "rubble pile" asteroid. The same trend will be also observed in Apophis and in the Aten-case figures if the lead time would have been larger.

Table 7 Approximate fragment population for a barely catastrophic fragmentation triggered with $100 \mathrm{~J} / \mathrm{kg}$.

\begin{tabular}{ccc}
\hline \hline Representative Mass & N Apophis & N Aten and Apollo cases \\
& $\mathrm{f}_{\mathrm{r}}=0.50$ & $\mathrm{f}_{\mathrm{r}}=0.50$ \\
\hline Largest Fragment & $2.45 \times 10^{10} \mathrm{~kg}$ & $4.6 \times 10^{10} \mathrm{~kg}$ \\
$1 \times 10^{10} \mathrm{~kg}$ & 0 & 0 \\
$5 \times 10^{9} \mathrm{~kg}$ & 0 & 0 \\
$1 \times 10^{9} \mathrm{~kg}$ & 0 & 0 \\
$5 \times 10^{8} \mathrm{~kg}$ & 0 & 0 \\
$1 \times 10^{8} \mathrm{~kg}$ & 1 & 2 \\
\hline \hline
\end{tabular}



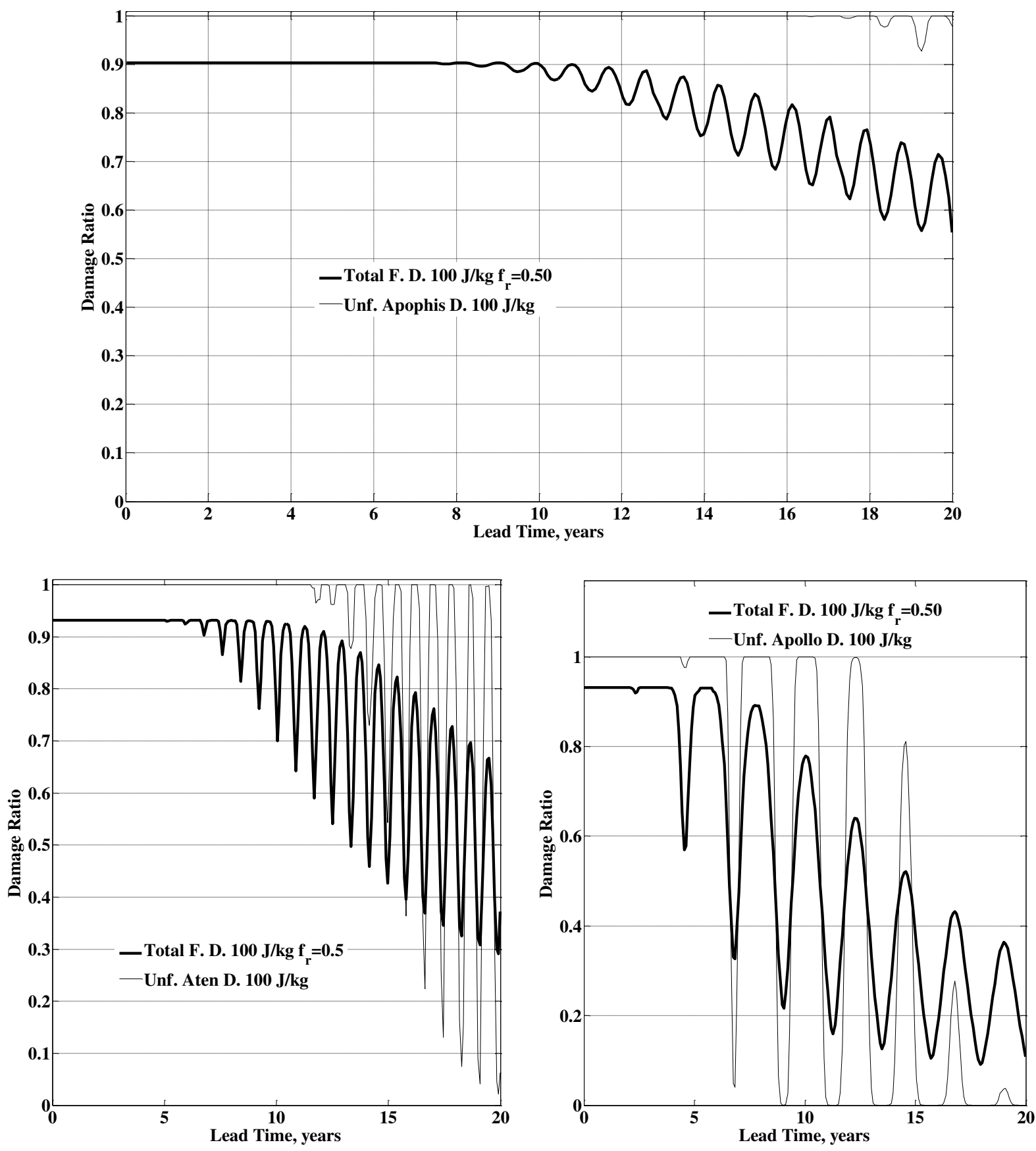

Fig. 17 Total fragmentation damage ratios of Apophis, Aten and Apollo-cases for a 100J/kg break-up: fragmented (thick black line) and unshattered case (thin black line) with a 50\% $3 \sigma$ in the delta-velocity.

\section{Catastrophic fragmentations with $1000 \mathrm{~J} / \mathrm{kg}$}

If energies of around $1000 \mathrm{~J} / \mathrm{kg}$ are provided, most of the mass escapes re-accumulation, thus increasing the population of fragment for all sizes. At this energy, several large and dangerous fragments should be expected. The damage probability ratio decreases with increasing lead time and reaches, with 20 years of lead time, levels of potential risk that are approximately half of those achieved through a deflection with $500 \mathrm{~J} / \mathrm{kg}$ (see Fig. 18). Perhaps the most important drawback of this energy level is the fact that a secondary attempt of deflection will have to deal not only with one dangerous fragment but with 3 to 6 . On top of that, at this level of collisional energy and by the 
results on fragmentation energies from Fig. 1, it seems clear that the most probable outcome of such an impulsive mitigation attempt would be a catastrophic fragmentation as described in Table 8 .

Table 8 Approximate fragment population for three catastrophic fragmentations triggered with $1000 \mathrm{~J} / \mathrm{kg}$.

\begin{tabular}{|c|c|c|c|c|c|c|}
\hline Representative Mass & \multicolumn{3}{|c|}{$\begin{array}{c}\mathrm{N} \\
\text { Apophis }\end{array}$} & \multicolumn{3}{|c|}{$\begin{array}{c}\mathrm{N} \\
\text { Aten and Apollo cases }\end{array}$} \\
\hline $\mathrm{f}_{\mathrm{r}}=$ & 0.50 & 0.25 & 0.10 & 0.50 & 0.25 & 0.10 \\
\hline $\begin{array}{c}\text { Largest Fragment } \\
{[\mathrm{kg}]}\end{array}$ & $1.75 \times 10^{10}$ & $9.15 \times 10^{9}$ & $2.93 \times 10^{9}$ & $3.5 \times 10^{10}$ & $1.93 \times 10^{10}$ & $6.06 \times 10^{9}$ \\
\hline $5 \times 10^{9} \mathrm{~kg}$ & 1 & 1 & 1 & 1 & 1 & 1 \\
\hline $1 \times 10^{9} \mathrm{~kg}$ & 2 & 2 & 2 & 3 & 4 & 3 \\
\hline $5 \times 10^{8} \mathrm{~kg}$ & 6 & 7 & 6 & 8 & 12 & 10 \\
\hline $1 \times 10^{8} \mathrm{~kg}$ & 11 & 15 & 12 & 16 & 24 & 21 \\
\hline
\end{tabular}



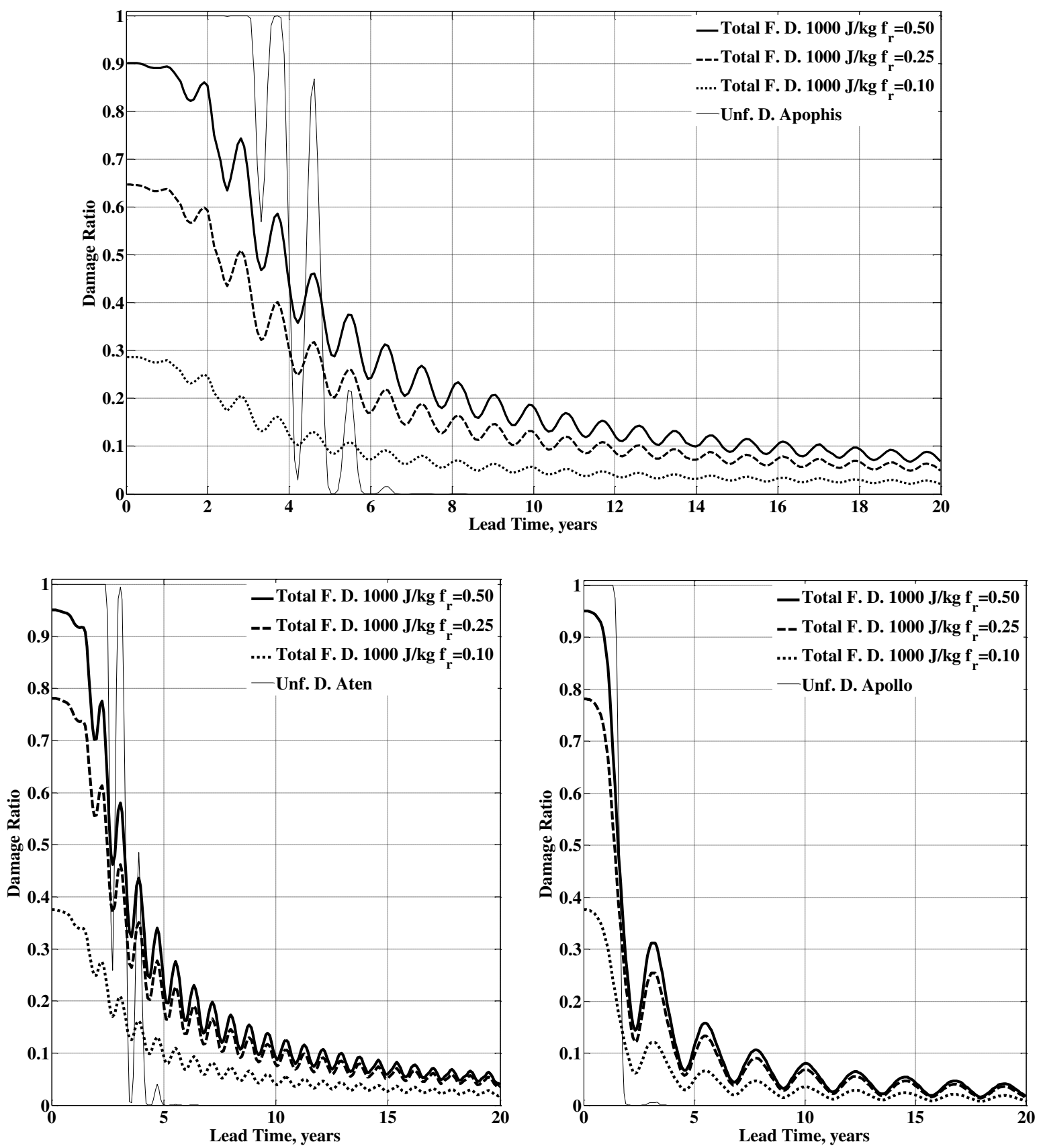

Fig. 18. Total fragmentation damage ratios of Apophis, Aten and Apollo-cases for a $1000 \mathrm{~J} / \mathrm{kg}$ break-up: fragmented (thick black lines) and unshattered cases (thin black line) with a $50 \% 3 \sigma$ in the deltavelocity.

Highly catastrophic fragmentations with $5000 \mathrm{~J} / \mathrm{kg}$

With a five-fold increase of the upper fragmentation limit considered in this work, the fragmentation should be expected to be highly catastrophic (see Table 9. The fragmentation level is clearly unknown, although, as seen by the fragmentations with $\mathrm{f}_{\mathrm{r}}=0.25$ and $\mathrm{f}_{\mathrm{r}}=0.10$ in Fig. 19 , a very high dispersion should be expected, achieving very low damage ratio for long lead times, which in some cases could be deemed negligible. However, although the unfragmented case seems highly improbable to exist at this level of energy, we should notice that the unfragmented option still represent a save way of achieving zero potential risk only after very short period. 
Table 9 Approximate fragment population for two highly catastrophic fragmentations triggered with 5000 $\mathrm{J} / \mathrm{kg}$.

\begin{tabular}{|c|c|c|c|c|}
\hline \multirow[t]{2}{*}{ Representative Mass } & \multicolumn{2}{|c|}{$\begin{array}{c}\mathrm{N} \\
\text { Apophis }\end{array}$} & \multicolumn{2}{|c|}{$\begin{array}{c}\mathrm{N} \\
\text { Aten and Apollo }\end{array}$} \\
\hline & $\mathrm{f}_{\mathrm{r}}=0.25$ & $\mathrm{f}_{\mathrm{r}}=0.10$ & $\mathrm{f}_{\mathrm{r}}=0.25$ & $\mathrm{f}_{\mathrm{r}}=0.10$ \\
\hline Largest Fragment & $5.7 \times 10^{9} \mathrm{~kg}$ & $2.29 \times 10^{9} \mathrm{~kg}$ & $1.1 \times 10^{10} \mathrm{~kg}$ & $4.37 \times 10^{9} \mathrm{~kg}$ \\
\hline $1 \times 10^{10} \mathrm{~kg}$ & 0 & 0 & 1 & 0 \\
\hline $5 \times 10^{9} \mathrm{~kg}$ & 1 & 1 & 2 & 1 \\
\hline $1 \times 10^{9} \mathrm{~kg}$ & 4 & 2 & 6 & 4 \\
\hline $5 \times 10^{8} \mathrm{~kg}$ & 9 & 6 & 15 & 11 \\
\hline $1 \times 10^{8} \mathrm{~kg}$ & 17 & 13 & 28 & 23 \\
\hline
\end{tabular}

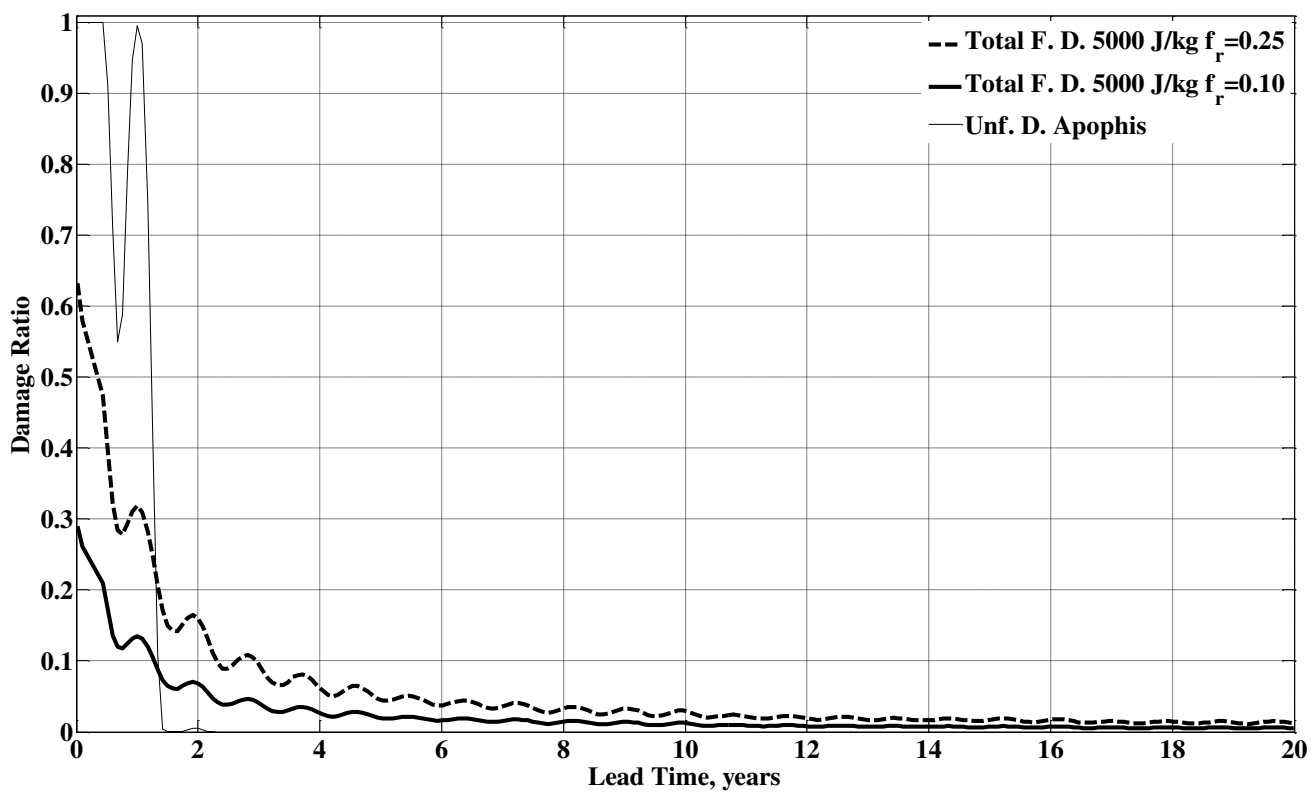



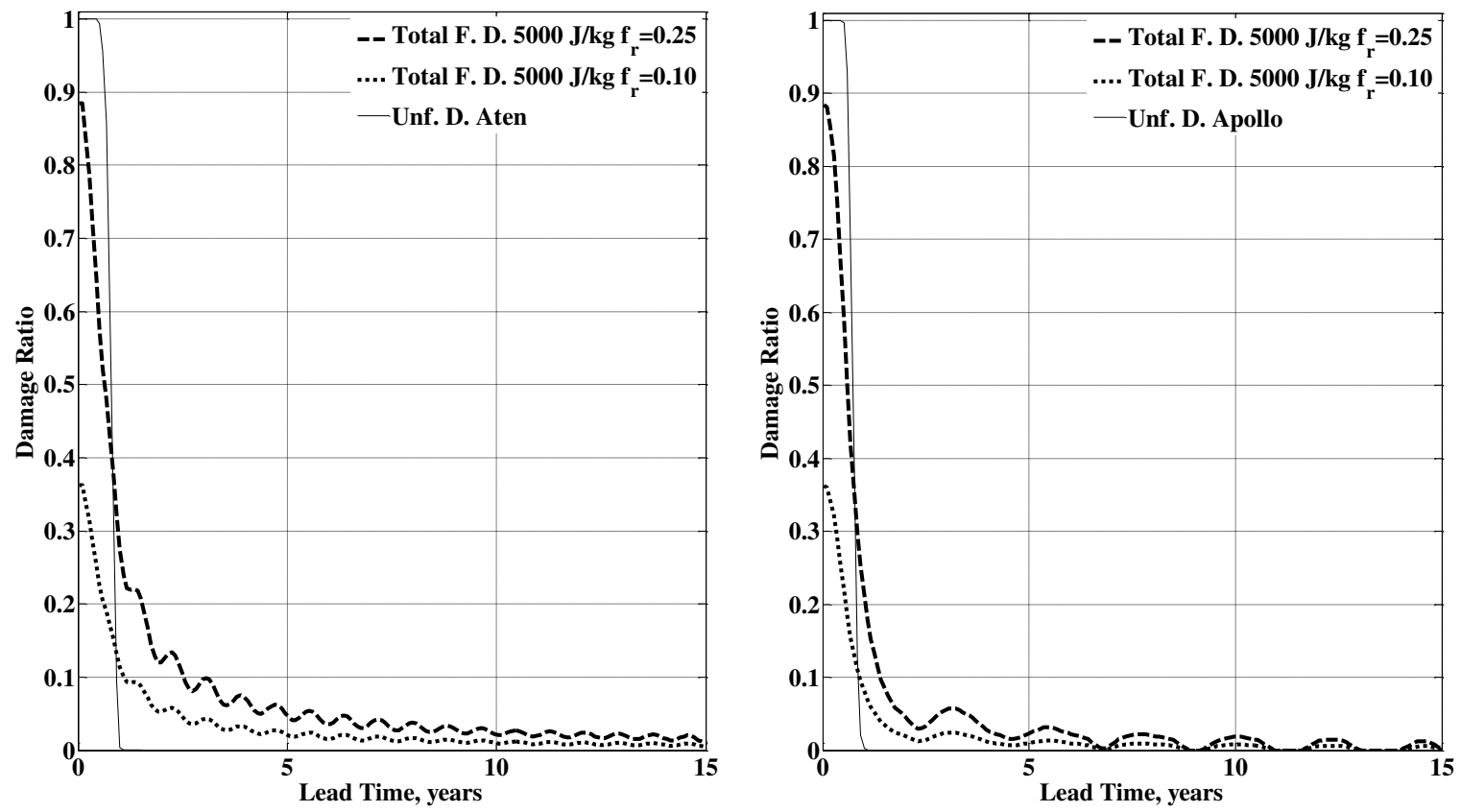

Fig. 19 Damage ratios of Apophis, Aten and Apollo-cases for a $5000 \mathrm{~J} / \mathrm{kg}$ break-up: fragmented (thick black lines) and unshattered case (thin black line) with a $50 \% 3 \sigma$ in the delta-velocity.

\section{Conclusions}

The work described in this paper examined the risk of fragmentation that impulsive asteroid deflection missions, such as the kinetic impactor or the nuclear interceptor, can cause when attempting to deflect an asteroid in a single impulsive maneuver. The levels of collisional energy required to break-up an asteroid were first estimated and, then, a fragmentation and dispersion model was introduced. The model was then used to analyze the evolution of fragments for up to 20 years after the break-up of the asteroid. Using the Earth impact probability of five different fragment sizes together with the approximate area that could be destroyed by each one fragments analyzed, the consequences of a fragmentation were estimated for several illustrative examples.

The results show that the energies required for a single impulsive deflection maneuver, i.e., those of a kinetic impactor or a nuclear interceptor, are dangerously close to the energies required to catastrophically disrupt an asteroid. Even for relatively large lead times, more than 10 years prior to the collision, the risk of fragmentation seems still considerable. We should also bear in mind that even if, instead of a single maneuver, several smaller impulses are given to the asteroid, in order to avoid surpassing the catastrophic fragmentation level, the material strength of the asteroid will decrease at each small impact, and the risk of fragmentation will not disappear, even if it may be considerably reduced. On the other hand, it is clear that if the impact aims only at a small deflection, to avoid a keyhole for example, a fragmentation is unlikely to happen.

If, instead, an undesired fragmentation of the threatening object occurs, it may result in a substantial increase of the damage to Earth. Considering that an undesired fragmentation occurs when applying collisional energies ranging from $100 \mathrm{~J} / \mathrm{kg}$ to $1000 \mathrm{~J} / \mathrm{kg}$, we can distinguish three different trends. For short lead times, the damage probability spawned by the fragmented asteroids is lower than the one of the unshattered object. This occurs not because some fragments miss the Earth, but as a result of the fragments that are too small to yield any noticeable damage at the surface of the Earth. Clearly, the maximum fragmented damage is strongly related with the fragmentation ratio resulting from a particular break-up. For medium lead times, i.e. those values at which the unfragmented scenario begins the transition from a damage ratio of 1 down to 0 , all the fragmented scenarios still retain a damage probability ratio that is considerably high, going approximately from 0.5 down to 0.15 . Finally, for very long lead times, the fragments disperse enough such that the damage becomes negligible. The time required to reach this point depends on the collisional energy used in the deflection attempt, but in any of the undesired fragmentation scenarios, the required lead time is longer than 20 years. 
Applying collisional energies much higher than $1000 \mathrm{~J} / \mathrm{kg}$ will result in highly catastrophic fragmentation. This level of energy in combination with lead times longer than ten years, may be used to fragment and disperse an asteroid to such a level that the potential damage becomes almost negligible. Although, for such a long lead time, both kinetic impulses lower than the fragmentation limit and low thrust deflection techniques are efficient options.

\section{References}

[1] L.W. Alvarez, W. Alvarez, F. Asaro and H.V. Michel, "Extraterrestrial Cause for the Cretaceous-Tertiary Extinction", Science, Vol. 208, No. 4448, June 1980, pp. 1095-1108. Doi: 10.1126/science.208.4448.1095

[2] K.A. Holsapple, "The Scaling of Impact Processes in Planetary Science", Annual Review of Earth and Planetary Science, Vol. 21, 1993, pp. 333-373. Doi: 10.1146/annurev.ea.21.050193.002001

[3] E.V. Ryan and H.J. Melosh, "Impact Fragmentation: From the Laboratory to Asteroids", Icarus, Vol. 133, No. 1, Apr. 1998, pp. 1-24. Doi: 10.1006/icar.1998.5915

[4] K.R. Housen and K.A. Holsapple, "On the Fragmentation of Asteroids and Planetary Satellites", Icarus, Vol. 84, 1990, pp. 226-253. Doi: 10.1016/0019-1035(90)90168-9

[5] H.J. Melosh, I.V. Nemchinov and Yu.I. Zetzer, "Non-nuclear Strategies for Deflecting Comets and Asteroids", Hazard Due to Comets and Asteroids, edited by T.Gehrels University of Arizona, Tucson, 1994, pp. 1110-1131.

[6] V.V. Ivashkin and V.V. Smirnov, "An Analysis of Some Methods of Asteroid Hazard Mitigation for the Earth", Planetary and Space Science, Vol. 43, No. 6, Nov. 1994, pp. 821-825. Doi: 10.1016/0032-0633(94)00225-G

[7] J.L.Remo, "Energy Requirements and Payload Masses for Near-Earth Objects Hazard Mitigation", Acta Astronautica, Vol. 47, No. 1, 2000, pp. 35-50. Doi: 10.1016/S0094-5765(00)00008-4

[8] "Near-Earth Objects Survey and Deflection Analysis of Alternatives", National Aeronautics and Space Administration, NASA Authorization Act of 2005, Mar. 2007.

[9] J.P. Sanchez, C. Colombo, M. Vasile and G. Radice, "Multi-criteria Comparison among Several Mitigation Strategies for Dangerous Near Earth Objects", Journal of Guidance, Control and Dynamics, Vol. 32, No. 1, Feb. 2009, pp. 121142. Doi: $10.2514 / 1.36774$

[10] T.J. Ahrens and A.W. Harris, "Deflection and Fragmentation of Near-Earth Asteroids", Nature, Vol. 360, Dec. 1992, pp. 429-433. Doi: 10.1038/360429a0

[11] D.R. Davis and E.V. Ryan, "On Collisional Disruption: Experimental Results and Scaling Laws", Icarus, Vol. 83, No. 156, 1990, pp. 182. Doi: 10.1016/0019-1035(90)90012-X

[12] R. Greenberg, J.F. Wacker, W.K. Hartmann and C.R. Chapman, "Planetesimal to Planets: Numerical Simulations of Collisional Evolution", Icarus, Vol. 35, July 1978, pp. 1-26. Doi: 10.1016/0019-1035(78)90057-X

[13] D.P. O'Brien and R. Greenberg, "Steady-state size distributions for collisional populations: analytical solution with size-dependent strength", Icarus, Vol. 164, Apr. 2003, pp. 334-345. Doi: 10.1016/S0019-1035(03)00145-3

[14] W.Wiesel, "Fragmentation of Asteroids and Artificial Satellites in Orbit", Icarus, Vol. 34, Apr. 1978, pp. 99-116. Doi: 10.1016/0019-1035(78)90130-6

[15] Duncan Steel, "Tunguska at 100", Nature, Vol. 453, June 2008, pp. 1157-1159. Doi: 10.1038/4531157a

[16] J.G. Hills and M.P. Goda, "The Fragmentation of Small Asteroids in the Atmosphere", The Astronomical Journal, Vol. 105, No. 3, Mar. 1993, pp. 1114-1144. Doi: 10.1086/116499

[17] P.A. Bland and N.A. Artemieva, "Efficient Disruption of Small Asteroids by Earth's Atmosphere", Nature, Vol. 424, July 2003, pp. 288-291. Doi: 10.1038/nature01757 
[18] S.R. Chesley and S.N. Ward, "A Quantitative Assessment of the Human and Economic Hazard from Impact-generated Tsunami", Natural Hazards, Vol. 38, 2006, pp. 355-374. Doi: 10.1007/s11069-005-1921-y

[19] K.A. Holsapple, "Catastrophic disruptions and cratering of solar system bodies: a review and new results", Planetary and Space Science, Vol. 42, No. 12, May 1994, pp. 1067-1078. Doi: 10.1016/0032-0633(94)90007-8

[20] G.H. Stokes, D.K. Yeomans, "Study to Determine the Feasibility of Extending the Search for Near-Earth Objects to Smaller Limiting Diameters", NASA, Aug. 2003.

[21] A.W.Harris, "The Rotation Rates of Very Small Asteroids: Evidence for 'Rubble Pile' Structure," Lunar and Planetary Science, Vol. 27, 1996, pp. 493.

[22] M. Vasile and C. Colombo, "Optimal Impact Strategies for Asteroid Deflection", Journal of Guidance, Control and Dynamics, Vol. 31, No. 4, 2008, pp. 858-872. Doi: 10.2514/1.33432

[23] J.P. Sanchez, "Asteroid Hazard Mitigation: Deflection Models and Mission Analysis", Ph.D. Thesis, University of Glasgow, Glasgow, Jan. 2009.

[24] W.J. Tedeschi, J.L. Remo, J.F. Schulze and R.P. Young, "Experimental Hypervelocity Impact Effects on Simulated Planetesimal Materials", International Journal of Impact Engineering, Vol. 17, No. 4-6, 1995, pp. 837-848. Doi: 10.1016/0734-743X(95)99904-6

[25] C. McInnes, "Deflection of Near-Earth Asteroids by kinetics Energy Impacts from Retrograde Orbits", Planetary and Space Science, Vol. 52, No. 7, June 2004, pp. 587-590. Doi: 10.1016/j.pss.2003.12.010

[26] A.E. Petropoulos, T.D. Kowalkowski, M.A. Vavrina, D.W. Parcher, P.A. Finlayson, G.J. Whiffen and J.A.Sims, "1stACT global trajectory optimisation competition: Results found at the Jet Propulsion Laboratory", Acta Astronautica, Vol. 61, No. 9, 2007, pp. 806-815. Doi: 10.1016/j.actaastro.2007.03.013

[27] H. Goldstein, Mecánica Clásica, 2nd ed., Reverté S.A. 1996, ISBN: 108429143068.

[28] D.E. Gault, E. M. Shoemaker and H.J. Moore, "Spray Ejected from the Lunar Surface by Meteoroid Impact", NASA Technical Note D-1767, 1963.

[29] A. Nakamura, K. Suguiyama and A. Fujiwara, "Velocity and Spin of Fragments from impact Distributions", Icarus, Vol. 100, No. 1, 1992, pp. 127-135. Doi: 10.1016/0019-1035(92)90023-Z

[30] W.B. Heard, "Dispersion of Ensembles of Non-Interacting Particles", Astrophysics and Space Science, Vol. 43, No. 1, Aug. 1976, pp. 63-82. Doi: 10.1007/BF00640556

[31] H. Mizutani, Y. Takagi and S.I. Kawakami, "New Scaling Laws on Impact Fragmentation", Icarus, Vol. 87, Oct. 1990, pp. 307-326. Doi: 10.1016/0019-1035(90)90136-W

[32] W. Benz and E. Asphaug, "Catastrophic Disruption Revised", Icarus, Vol. 142, No. 1, Nov. 1999, pp. 5-20. Doi: 10.1006/icar.1999.6204

[33] A.W. Harris, E.G. Fahnestock, and P. Pravec, "On the shapes and spins of "rubble pile" asteroids," Icarus, Vol. 199, No. 2, Feb. 2009, pp. 310-318. Doi: 10.1016/j.icarus.2008.09.012

[34] P. Pravec and A.W. Harris, "Binary asteroids population. 1. Angular momentum content", Icarus, Vol. 190, Apr. 2007, pp. 250-259. Doi: 10.1016/j.icarus.2007.02.023

[35] C.R. Chapman and D. Morrison, "Impacts on the Earth by asteroids and comets: assessing the hazard", Nature, Vol. 367, Jan. 1994, pp. 33-40. Doi: 10.1038/367033a0 